TRANSACTIONS OF THE

AMERICAN MATHEMATICAL SOCIETY

Volume 313, Number 2, June 1989

\title{
A CALCULUS APPROACH TO HYPERFUNCTIONS. II
}

\author{
TADATO MATSUZAWA
}

\begin{abstract}
We consider any hyperfunctions with the compact support as initial values of the solutions of the heat equation. The main aim of this paper is to unify the theory of distributions and hyperfunctions as well as simplify proofs of some important results via heat kernel.
\end{abstract}

\section{INTRODUCTION}

In the previous paper [9], we have discussed the theory of the hyperfunctions by a calculus approach motivated by the analysis developed in [3, Chapter 9]. We have shown in [9] the idea of our approach. Namely, we have proposed to take hyperfunctions with the compact support as initial values of the solutions of the heat equation. The main aim of this paper is to reformulate the foundation of hyperfunction theory by a limiting process via heat kernel in the theory of Schwartz distributions. For this purpose, two fundamental tools will be used. One is the estimate for the heat kernel in (1.14) which is obtained as a special case of the result given in [1, Chapter 4]. The other is a series of structure theorems on ultradistributions given in [5, 6 and 7]. As was mentioned in [9], the advantage of our method is that it can unify the theory of distributions and hyperfunctions as well as simplify proofs of some important results.

In $\S 1$, we briefly recall the definitions of distributions and hyperfunctions with the compact support. Especially, the space of hyperfunctions (analytic functionals) $A^{\prime}[K]$ with the compact support in $K \subset R^{n}(n \geq 1)$ is considered as the dual of the space $A[K]$ of functions which are real analytic near $K$. We will prepare a useful estimate for the heat kernel in Proposition 1.1. In $\S 2$, we will prove that we can take $u \in A^{\prime}[K]$ as an initial value of a unique solution $U$ of the heat equation

$$
\left\{\begin{array}{l}
(\partial / \partial t-\Delta) U(x, t)=0 \quad \text { in } R_{+}^{n+1}=R^{n} \times R_{+}, \\
U(\cdot, 0)=u
\end{array}\right.
$$

We shall see in Theorem 2.1 that Schwartz distributions and ultradistributions are also characterized by the asymptotic behavior of $U(\cdot, t)$ as $t \rightarrow 0$ at the

Received by the editors June 8, 1987 and, in revised form, December 8, 1987.

1980 Mathematics Subject Classification (1985 Revision). Primary 46F15; Secondary 46F05, 46F10, 46F12, 35K05. 
same time. At the end of $\S 2$, we shall apply this characterization to give a simple proof for a local existence theorem to the equation

$$
P(D) u=f \quad \text { in } \Omega
$$

in the hyperfunction space $B(\Omega)$, where $\Omega$ is any bounded open set in $R^{n}$ and $P(D)$ is a partial differential operator with constant coefficients. In $\S 3$, we shall show in Theorem 3.1 that the local regularity property of an element $u \in A^{\prime}[K]$ is also described by the asymptotic behavior of $U(\cdot, t)$ as $t \rightarrow 0$. In $\S 4$, we formulate a completeness result on hyperfunctions in Theorem 4.1 which will play a dominant role in the microlocal calculus developed in the last section. This theorem corresponds to Theorem 9.1.7 in [3]. As suggested by the results given in $\S 3$, we shall give, in $\S 5$, the definition of wave front set which is related to the asymptotic behavior of $U(\cdot, t)$ as $t \rightarrow 0$. We hope to clarify the Bros-Iagolnitzer definition of wave front set on the basis of our results obtained so far.

Further investigation of generalized functions will be made in a forthcoming paper.

The author expresses his profound gratitude to M. Miyake and Y. Morimoto for valuable discussions during the preparation of this paper.

\section{SPACES OF DISTRIBUTIONS AND HYPERFUNCTIONS}

Let $\Omega$ be an open subset of $R^{n}$ whose points are denoted by $x=\left(x_{1}, \ldots\right.$, $\left.x_{n}\right)$. We use general notations such as $|\alpha|=\alpha_{1}+\cdots+\alpha_{n}$ for a multi-index $\alpha=\left(\alpha_{1}, \ldots, \alpha_{n}\right)$ and $D^{\alpha}=D_{1}^{\alpha_{1}} \cdots D_{n}^{\alpha_{n}}, D_{j}=-i \partial / \partial x_{j}, j=1, \ldots, n$, etc.

Definition 1.1. Let $\phi \in C^{\infty}(\Omega)$. Then we say that $\phi$ is in $\mathscr{E}^{\{s\}}(\Omega)$ for $s>1$ if for any compact subset $K$ of $\Omega$ there are positive constants $C_{0}$ and $C_{1}$ such that

$$
\sup _{x \in K}\left|D^{\alpha} \phi(x)\right| \leq C_{0} C_{1}^{|\alpha|} \alpha !^{s} .
$$

We say also that $\phi$ is in $\mathscr{E}^{(s)}(\Omega)$ for $s>1$ if for any compact subset $K$ of $\Omega$ and for any positive number $h$ there is a constant $C$ such that

$$
\sup _{x \in K}\left|D^{\alpha} \phi(x)\right| \leq C h^{|\alpha|} \alpha !^{s} \text {. }
$$

We denote by $\mathscr{D}^{\{s\}}(\Omega)$ and $\mathscr{D}^{(s)}(\Omega)$ the subspaces of $\mathscr{E}^{\{s\}}(\Omega)$ and $\mathscr{E}^{(s)}(\Omega)$ respectively which consist of functions with compact support in $\Omega$.

It is well known that the function

$$
f(x)=\left\{\begin{array}{ll}
\exp \left[-x^{1 /(s-1)}\right], & x>0 \\
0, & x \leq 0
\end{array}(s>1)\right.
$$

is in $\mathscr{E}^{\{s\}}(R)$ but not in $\mathscr{E}^{(s)}(R)$. Thus we have the inclusion

$$
\begin{array}{ll}
\mathscr{E}^{(s)}(\Omega) \varsubsetneqq \mathscr{E}^{\{s\}}(\Omega) \varsubsetneqq \mathscr{E}^{(t)}(\Omega), & 1<s<t<\infty, \\
\mathscr{D}^{(s)}(\Omega) \varsubsetneqq \mathscr{D}^{\{s\}}(\Omega) \varsubsetneqq \mathscr{D}^{\{t\}}(\Omega), & 1<s<t<\infty .
\end{array}
$$


The topology of such spaces is defined as follows:

(i) We say a sequence $\left\{f_{j}(x)\right\} \subset \mathscr{E}^{\{s\}}(\boldsymbol{\Omega})$ converges to zero in $\mathscr{E}^{\{s\}}(\boldsymbol{\Omega}), s>$ 1 , if for any compact set $K$ of $\Omega$ there is a constant $C$ such that

$$
\sup _{x \in X} \frac{\left|D^{\alpha} f_{j}(x)\right|}{C^{|\alpha|} \alpha !^{s}} \rightarrow 0 \quad \text { as } j \rightarrow \infty .
$$

(ii) We say a sequence $\left\{f_{j}(x)\right\} \subset \mathscr{E}^{(s)}(\Omega)$ converges to zero in $\mathscr{E}^{(s)}(\Omega), s>$ 1 , if for any compact set $K$ of $\Omega$ and for any $h>0$ we have

$$
\sup _{x \in K} \frac{\left|D^{\alpha} f_{j}(x)\right|}{h^{|\alpha|} \alpha !^{s}} \rightarrow 0 \text { as } j \rightarrow \infty .
$$

(iii) We say a sequence $\left\{f_{j}(x)\right\} \subset \mathscr{D}^{\{s\}}(\Omega)$ (or $\mathscr{D}^{(s)}(\Omega)$ ) converges to zero in $\mathscr{D}^{\{s\}}(\Omega)$ (or in $\mathscr{D}^{(s)}(\Omega)$ ), s>1, if there is a compact set $K$ of $\Omega$ such that $\operatorname{supp} f_{j} \subset K, j=1,2, \ldots$, and $f_{j} \rightarrow 0$ in $\mathscr{E}^{\{s\}}(\Omega)$ (or in $\mathscr{E}^{(s)}(\Omega)$ ).

As usual, we denote by $\mathscr{D}^{\{s\}^{\prime}}(\Omega)$ (by $\mathscr{D}^{(s)^{\prime}}(\Omega), \mathscr{E}^{\{s\}^{\prime}}(\Omega)$ and $\mathscr{E}^{(s)^{\prime}}(\Omega)$ ) the strong dual space of $\mathscr{D}^{\{s\}}(\Omega)$ (of $\mathscr{D}^{(s)}(\Omega), \mathscr{E}^{\{s\}}(\Omega)$ and $\mathscr{E}^{(s)}(\Omega)$ respectively) and call its elements ultradistributions on $\Omega$.

We have the inclusion

$$
\begin{gathered}
\mathscr{D}^{\prime}(\boldsymbol{\Omega}) \subset \mathscr{D}^{\{s\}^{\prime}}(\boldsymbol{\Omega}) \subset \mathscr{D}^{(s)^{\prime}}(\boldsymbol{\Omega}), \quad s>1, \\
\mathscr{E}^{\prime}(\boldsymbol{\Omega}) \subset \mathscr{\mathscr { E }}^{\{s\}^{\prime}}(\boldsymbol{\Omega}) \subset \mathscr{E}^{(s)^{\prime}}(\boldsymbol{\Omega}) \subset \mathscr{D}^{(s)^{\prime}}(\boldsymbol{\Omega}), \quad s>1,
\end{gathered}
$$

where $\mathscr{D}^{\prime}(\Omega)$ and $\mathscr{E}^{\prime}(\Omega)$ are the spaces of Schwartz distributions. It is well known that $\mathscr{E}^{\{s\}^{\prime}}(\Omega)$ consists of ultradistributions with compact support in $\Omega$ for $s>1$ like the space $\mathscr{E}^{\prime}(\Omega)$. For further details we refer the reader to $[5,6$ and 7]. Let $K \subset R^{n}$ be a compact set. We denote by $\mathscr{E}_{K}^{\prime}$ and $\mathscr{E}_{K}^{\{s\}^{\prime}}, 1<s<\infty$, the set of Schwartz and ultradistributions with supports in $K$ respectively.

Next we shall define the spaces of analytic functionals along the lines of [ 3 and 9]. Let $K$ be a compact subset of $R^{n}$.

Definition 1.2. We say that $\phi(x)$ is in $A[K]$ if $\phi(x)$ is real analytic in a neighborhood of $K$ and there are positive constants $C_{0}$ and $C_{1}$ such that

$$
\sup _{x \in K}\left|D^{\alpha} \phi(x)\right| \leq C_{0} C_{1}^{|\alpha|} \alpha ! .
$$

We say $\phi_{j} \rightarrow 0$ in $A[K]$ as $j \rightarrow \infty$ if there is a constant $h>0$ such that

$$
\sup _{\substack{x \in K \\ \alpha}} \frac{\left|D^{\alpha} \phi_{j}(x)\right|}{h^{|\alpha|} \alpha !} \rightarrow 0 \text { as } j \rightarrow \infty .
$$

We denote by $A^{\prime}[K]$ the strong dual space of $A[K]$ and call its elements analytic functionals carried by $K$.

We have changed the notation in [3 and 9] slightly and use $A[K]$ in place of $A(K)$. We denote by $A$ the space of entire functions in $\mathbf{C}^{n}$ in the following. 
Theorem 1.1 (cf. [5 and 9]). Let $u \in A^{\prime}[K]$. Then for any $h>0$ there exists $a$ constant $C=C(h, u)$ such that

$$
|u(\phi)| \leq C \sup _{x \in K} \frac{\left|D^{\alpha} \phi(x)\right|}{h^{|\alpha|} \alpha !}, \quad \phi \in A .
$$

This is equivalent to the condition that for every complex neighborhood $\omega$ of $K$

$$
|u(\phi)| \leq C_{\omega} \sup |\phi|, \quad \phi \in A
$$

Let $u \in \mathscr{E}^{\{s\}^{\prime}}\left(R^{n}\right)\left(\mathscr{E}^{(s)^{\prime}}\left(R^{n}\right)\right)$ with $s>1$. Then there is a compact set $K \subset R^{n}$ such that for any $h>0$ there is a constant $C>0$ (there are constants $h$ and $C$ ) satisfying

$$
(1.11)_{s} \quad|u(\phi)| \leq C \sup _{x \in K} \frac{\left|D^{\alpha} \phi(x)\right|}{h^{|\alpha|} \alpha !^{s}}, \quad \phi \in \mathscr{E}^{\{s\}}\left(R^{n}\right) \quad\left(\phi \in \mathscr{E}^{(s)}\left(R^{n}\right)\right) .
$$

We can consider $A^{\prime}\left[K_{1}\right] \subset A^{\prime}\left[K_{2}\right]$ if $K_{1} \subset K_{2}$ and set $A^{\prime}=\bigcup_{K} A^{\prime}[K]$. Then we have the inclusion

$$
\mathscr{E}^{\prime}\left(R^{n}\right) \subset \mathscr{E}^{\{s\}^{\prime}}\left(R^{n}\right) \subset \mathscr{E}^{(s)^{\prime}}\left(R^{n}\right) \subset A^{\prime}, \quad 1<s<\infty .
$$

We denote by $E(x, t)$ the $n$-dimensional heat kernel:

$$
E(x, t)= \begin{cases}(4 \pi t)^{-n / 2} \exp \left[-|x|^{2} / 4 t\right], & t>0 \\ 0, & t<0\end{cases}
$$

Proposition 1.1 (cf. [1, Chapter 4]). $E(\cdot, t)$ is an entire function of order 2 for every $t>0$. We have the following properties on $E$ :

(i) We have

$$
\int_{R^{n}} E(x, t) d x=1, \quad t>0 ;
$$

(ii) There are positive constants $C$ and $a$ such that

$$
\left|D_{x}^{\alpha} E(x, t)\right| \leq C^{|\alpha|} t^{-(n+|\alpha|) / 2} \alpha !^{1 / 2} \exp \left[-a|x|^{2} / 4 t\right], \quad t>0,
$$

where a can be taken as close as desired to 1 and $0<a<1$.

Proof. (i) We omit the well-known calculus to prove (1.13).

(ii) We shall prove (1.14) in the case $n=1$ for simplicity. The derivatives of $E(x, t)$ can be evaluated by means of the Cauchy formula

$$
\partial_{x}^{k} E(x, t)=\frac{k !}{2 \pi i} \int_{\Gamma_{R}} \frac{E(z, t)}{(z-x)^{k+1}} d z,
$$

where $\Gamma_{R}$ is a circle of radius $R$ in $\mathbf{C}$ with center at the point $x$. From (1.15) we find

$$
\left|\partial_{x}^{k} E(x, t)\right| \leq \frac{k !}{\sqrt{4 \pi t} R^{k}} \exp \left[\left(-\bar{x}^{2}+R^{2}\right) / 4 t\right], \quad R>0
$$


where $\bar{x}=x-R, 0$ or $x+R$. Let us select the radius $R$ so that the ratio $\exp \left[R^{2} / 4 t\right] / R^{k}$ would attain its minimum. This is realized for $R=\sqrt{2 k t}$, so that $(1.16)$ reduces to

$$
\left|\partial_{x}^{k} E(x, t)\right| \leq \frac{1}{\sqrt{4 \pi}}(e / 2)^{k / 2} t^{-(1+k) / 2} k ! k^{-(k / 2)} \exp \left[-\bar{x}^{2} / 4 t\right] .
$$

The last factor may be estimated as follows. Let us replace $\bar{x}$ by $x+\theta R$, where $|\theta| \leq 1$; then

$$
\exp \left[-\bar{x}^{2} / 4 t\right]=\exp \left[-(x+\theta R)^{2} / 4 t\right] \leq \exp \left[-a x^{2} / 4 t\right] \exp \left[a^{\prime} R^{2} / 4 t\right],
$$

where the constant $a$ may be chosen so that it is as close as desired to 1 and $0<a<1$. Furthermore $R^{2}=2 k t$ hence we have

$$
\left|\partial_{x}^{k} E(x, t)\right| \leq \frac{1}{\sqrt{4 \pi}} e^{\left(1+a^{\prime}\right) k / 2} t^{-(k / 2)} k !^{1 / 2} \exp \left[-a x^{2} / 4 t\right],
$$

from which we obtain (1.14) in case $n=1$.

Proposition 1.2. For every $\phi \in A[K]$, let

$$
\phi_{t}(x)=\int E(x-y, t) \chi(y) \phi(y) d y, \quad t>0,
$$

where $\chi \in C_{0}^{\infty}\left(R^{n}\right)$ such that $\chi=1$ in a neighborhood of $K$. Then $\phi_{t} \rightarrow \phi$ in $A[K]$ as $t \rightarrow 0_{+}$. Also for every $\psi \in \mathscr{D}^{\{s\}}\left(R^{n}\right)\left(\mathscr{D}^{(s)}\left(R^{n}\right)\right)$ with $s>1$, let

$$
\psi_{t}(x)=\chi(x) \int E(x-y, t) \psi(y) d y,
$$

where $\chi \in \mathscr{D}^{\{s\}}\left(R^{n}\right), \chi=1$ in a neighborhood of $\operatorname{supp} \psi$. Then $\psi_{t} \rightarrow \psi$ in $\mathscr{D}^{\{s\}}\left(R^{n}\right)\left(\mathscr{D}^{(s)}\left(R^{n}\right)\right)$ as $t \rightarrow 0_{+}$.

Proof. Let $\phi \in A[K]$. Then there are positive constants $C, h$ and $\delta$ such that

$$
\sup _{x \in K_{\delta}}\left|D^{\alpha} \phi(x)\right| \leq C h^{|\alpha|} \alpha !,
$$

where $K_{\delta}=\left\{x \in R^{n} ; \operatorname{dis}(x, K) \leq \delta\right\}$. We may consider $\chi=1$ in a neighborhood of $K_{\delta}$. By integration by making use of (1.17), we have for $|y| \leq \delta$

$$
\sup _{x \in K}\left|D^{\alpha} \phi(x-y)-D^{\alpha} \phi(x)\right| \leq C|y| h^{|\alpha|+1}(|\alpha|+1) ! .
$$

Hence there are positive constants $C^{\prime}$ and $H$ such that

$$
\sup _{x \in K}\left|D^{\alpha} \phi(x-y)-D^{\alpha} \phi(x)\right| \leq C^{\prime}|y|(H h)^{|\alpha|}|\alpha| !, \quad|y| \leq \delta .
$$

On the other hand we have for $x \in K$

$$
\begin{aligned}
D_{x}^{\alpha}\left(\phi_{t}(x)-\phi(x)\right)= & \int_{|y| \leq \delta} E(y, t) D_{x}^{\alpha}(\phi(x-y)-\phi(x)) d y \\
& +D_{x}^{\alpha} \int_{|x-y| \geq \delta} E(x-y, t) \chi(y) \phi(y) d y \\
& -D_{x}^{\alpha} \phi(x) \int_{|x-y| \geq \delta} E(x-y, t) d y \\
= & I_{1, \alpha}+I_{2, \alpha}+I_{3, \alpha} .
\end{aligned}
$$


For any $\varepsilon>0$, taking $\delta$ so small that $C^{\prime} \delta^{n+1} \leq \varepsilon$, we have

$$
\left|I_{1, \alpha}\right| /(H h)^{|\alpha|}|\alpha| ! \leq \varepsilon \text {. }
$$

Next by using (1.14), we have the estimation of the form

$$
\begin{aligned}
\frac{\left|I_{2, \alpha}\right|}{(H h)^{|\alpha|}|\alpha| !} & \leq(C / H h)^{|\alpha|}\left(t^{-|\alpha|} /|\alpha| !\right)^{1 / 2} \exp \left[-c \delta^{2} / 2 t\right] \\
& \leq \exp \left[(1 / 2 t)\left(C / H h-c \delta^{2}\right)\right] \\
& \leq \exp \left[-c \delta^{2} / 4 t\right], \quad t>0,
\end{aligned}
$$

if we take $H$ so large that $C / H h \leq c \delta^{2} / 2$. By taking $H$ larger if necessary, we have

$$
\frac{\left|I_{3, \alpha}\right|}{(H h)^{|\alpha|}|\alpha| !} \leq \exp \left[-c \delta^{2} / 2 t\right] \text {. }
$$

Thus we have $\phi_{t} \rightarrow \phi$ in $A[K]$.

Now let $\psi \in \mathscr{D}^{\{s\}}\left(R^{n}\right)$ with $s>1$. Then there are constants $C$ and $h$ such that

$$
\sup _{x \in R^{n}}\left|D^{\alpha} \psi(x)\right|<C h^{|\alpha|} \alpha !^{s},
$$

and $\operatorname{supp} \psi$ is a compact set in $R^{n}$. Then similarly as before we have

$$
\sup _{x}\left|D^{\alpha} \psi(x-y)-D^{\alpha} \psi(x)\right| \leq C^{\prime}|y|(H h)^{|\alpha|}|\alpha| !^{s}, \quad y \in R^{n},
$$

and

$$
\begin{aligned}
D_{x}^{\alpha}\left(\psi_{t}(x)-\psi(x)\right)= & \int_{|y| \leq \delta} E(y, t) D_{x}^{\alpha}(\psi(x-y)-\psi(x)) d y \\
& +\int_{|y| \geq \delta} \sim d y \\
= & J_{1, \alpha}+J_{2, \alpha} .
\end{aligned}
$$

We can treat $J_{1, \alpha}$ the same way as in $I_{1, \alpha}$. By using (1.18) and by the fact that

$$
\int_{|y| \geq \delta} E(y, t) d y \rightarrow 0 \quad \text { as } t \rightarrow 0_{+},
$$

we see $\psi_{t} \rightarrow \psi$ in $\mathscr{D}^{\{s\}}\left(R^{n}\right)$

Similar arguments can be applied in case $\psi \in \mathscr{D}^{(s)}\left(R^{n}\right)$. Q.E.D.

\section{ChaRACTERIZATION OF HYPERFUNCTIONS AND DISTRIBUTIONS}

Let $u \in A^{\prime}[K]$. Then the function

$$
U(x, t)=u_{y}(E(x-y, t)), \quad x \in R^{n}, t>0,
$$

is well defined since $E(x-\cdot, t) \in A$ for every $(x, t) \in R_{+}^{n+1}=\{(x, t) ; x \in$ $\left.R^{n}, t>0\right\}$. 
Theorem 2.1 (cf. [9, Theorem 1.2]). (i) Let $u \in A^{\prime}[K]$. Then $U(x, t) \in$ $C^{\infty}\left(R_{+}^{n+1}\right)$ and $U(\cdot, t) \in A$ for each $t>0$. Furthermore $U$ satisfies the following conditions:

$$
(\partial / \partial t-\Delta) U(x, t)=0 \quad \text { in } R_{+}^{n+1} ;
$$

for every $\varepsilon>0$ we have

$$
|U(x, t)| \leq C_{\varepsilon} \exp \left[(\varepsilon / t)-\operatorname{dis}(x, K)^{2} / 4 t\right] \text { in } R_{+}^{n+1} ;
$$

$U(x, t) \rightarrow u$ as $t \rightarrow 0_{+}$in the following sense:

$$
u(\phi)=\lim _{t \rightarrow 0} \int_{\Omega} U(x, t) \phi(x) d x, \quad \phi \in A,
$$

where $\Omega$ is an arbitrary bounded neighborhood of $K$.

Conversely, every $C^{\infty}$-function defined in $R_{+}^{n+1}$ satisfying conditions (2.2) and (2.3) can be expressed in the form (2.1) with unique element $u \in A^{\prime}[K]$.

(ii) In case $u \in \mathscr{E}_{K}^{\{s\}^{\prime}}\left(\mathscr{E}_{K}^{(s)^{\prime}}\right)$ with $s>1$, condition (2.3) of the above assertion is replaced by the following:

For every $\varepsilon, \delta>0$ (there is a positive constant $C_{\varepsilon, \delta}$ such that) we have

$$
|U(x, t)| \leq C_{\varepsilon, \delta} \exp \left[(\varepsilon / t)^{1 /(2 s-1)}-\operatorname{dis}\left(x, K_{\delta}\right)^{2} / 8 t\right] \text { in } R_{+}^{n+1},
$$

where $K_{\delta}=\{x ; \operatorname{dis}(x, K) \leq \delta\}$.

(iii) In case $u \in \mathscr{E}_{K}^{\prime}$, condition (2.3) is replaced by the following:

There exists a nonnegative integer $N=N(u)$ such that

$$
|U(x, t)| \leq C_{\delta} t^{-N} \exp \left[-\operatorname{dis}\left(x, K_{\delta}\right)^{2} / 8 t\right] \text { in } R_{+}^{n+1} .
$$

In each case of (ii) and (iii) condition (2.4) is replaced by corresponding one:

$$
U(\cdot, t) \rightarrow u \text { in } \mathscr{E}^{\{s\}^{\prime}}\left(R^{n}\right)\left(\mathscr{E}^{(s)^{\prime}}\left(R^{n}\right), \mathscr{E}^{\prime}\left(R^{n}\right) \text { resp. }\right) .
$$

Proof. (i) The proof of this part was given in [9]. We shall reproduce it for completeness. Let $u \in A^{\prime}[K]$. Then we find obviously that the function $U(x, t)$ defined by (2.1) belongs to $C^{\infty}\left(R_{+}^{n+1}\right)$ and satisfies the heat equation (2.2). We take $z=y+i \eta, y, \eta \in R^{n}$, and observe that

$$
\operatorname{Re}-(x-y-i \eta)^{2}=-(x-y)^{2}+\eta^{2} .
$$

Then by applying (1.11) we have (2.3) if we take $|\eta|$ sufficiently small. Now let $\Omega \subset R^{n}$ be a bounded open set containing $K$ and let

$$
G(y, t)=\int_{\Omega} E(x-y, t) \phi(x) d x, \quad \phi \in A .
$$

Then by Proposition 1.1 and Proposition 1.2, we can easily see

$$
G(\cdot, t) \rightarrow \phi \text { in } A[K] \text { as } t \rightarrow 0_{+} .
$$

We have

$$
\int_{\Omega} U(x, t) \phi(x) d x=u_{y}(G(y, t))
$$


by taking limit of the Riemann sum of the left-hand side. Then by applying (2.7) we have (2.4).

Now we will prove the converse. We shall use some fundamental properties of ultradistributions for which we refer to [5 and 7]. Let $U(x, t)$ be a function defined in $R_{+}^{n+1}$ satisfying (2.2) and (2.3). Then by virtue of (2.3) we can extend $U$ beyond the hyperplane $t=0$ as an ultradistribution $\widetilde{U} \in \mathscr{D}^{\{2\}^{\prime}}\left(R^{n+1}\right)$ such that

$$
\tilde{U}(x, t)= \begin{cases}U(x, t), & t>0, \\ 0, & t<0 .\end{cases}
$$

This follows from a fundamental property of ultradistributions (cf. [7, Theorem 2.27]), and we shall find the idea of its proof in $\S 4$. We have

$$
\tilde{U} \in C^{\infty}\left(R^{n+1} \backslash(K \times\{0\})\right)
$$

and

$$
(\partial / \partial t-\Delta) \tilde{U}(x, t)=f \text { in } R^{n+1},
$$

where $f \in \mathscr{E}_{K \times\{0\}}^{\{2\}^{\prime}}$. Again by a fundamental property of ultradistributions with support in a hyperplane (cf. [7, Theorem 3.1]), $f$ is uniquely represented as

$$
f=\sum_{k=0}^{\infty} f_{k}(x) \otimes \delta^{(k)}(t)
$$

with $f_{k}(x) \in \mathscr{E}_{K}^{\{2\}^{\prime}}, k=0,1, \ldots$, satisfying the following conditions:

For every $L>0, h>0$ and $\delta>0$ there is a constant $C$ such that

$$
\left|f_{k}(\phi)\right| \leq C L^{k} k !^{-2} \sup _{x \in K_{\delta}} \frac{\left|D^{\alpha} \phi(x)\right|}{h^{|\alpha|} \alpha !^{2}}, \quad \phi \in \mathscr{E}^{\{2\}}\left(R^{n}\right),
$$

and

$$
\operatorname{supp} f=\overline{\bigcup_{k} \operatorname{supp} f_{k}} \text {. }
$$

We define an ultradistribution $v \in \mathscr{D}^{\{2\}^{\prime}}\left(R^{n+1}\right)$ as follows:

$$
v(x, t)= \begin{cases}\sum_{k=0}^{\infty} \int\left(\partial_{t}\right)^{k} E(x-y, t) f_{k}(y) d y, & t>0 \\ 0, & t<0\end{cases}
$$

That is, we define $v$ formally

$$
v(x, t)=\iint E(x-y, t-\tau) f(y, \tau) d y d \tau .
$$

Since we have

$$
(\partial / \partial t-\Delta) E(x-y, t-\tau)=\delta(x-y, t-\tau)
$$

also in the ultradistribution sense, we have

$$
(\partial / \partial t-\Delta) v(x, t)=f \quad \text { in } R^{n+1} .
$$


We will show that $\widetilde{U}=v$. First we have $\widetilde{U}-v \in \mathscr{D}^{\{2\}^{\prime}}\left(R^{n+1}\right)$ and

$$
(\partial / \partial t-\Delta)(\tilde{U}-v)=0 \text { in } R^{n+1} .
$$

Using relation (2.12) and the well-known fact that $E(x, t) \in \mathscr{E}_{x, t}^{\{1,2\}}\left(R^{n+1} \backslash 0\right)$, or by the Gevrey hypoellipticity for the heat equation proved in [11], we have

$$
\tilde{U}-v \in \mathscr{E}^{\{2\}}\left(R^{n+1}\right), \widetilde{U}-v=0, t<0 .
$$

Furthermore by applying (2.9) and Proposition 1.1 to (2.12) we have the estimation of type $(2.3)$ for $v(x, t)$. Thus we can easily show that $\tilde{U}-v$ is bounded in the whole space $R^{n+1}$. By the well-known uniqueness theorem for the solutions of the Cauchy problem to the heat equation we have $\widetilde{U}-v=0$. It remains to prove that there exists an element $v_{0} \in A^{\prime}[K]$ such that

$$
v(\cdot, t) \rightarrow v_{0} \text { in } A^{\prime}[K] \text { as } t \rightarrow 0_{+} .
$$

For every $t>0$, we have

$$
v(x, t)=\sum_{k=0}^{\infty} \int \Delta^{k} E(x-y, t) f_{k}(y) d y .
$$

Let $\Omega \subset R^{n}$ be a bounded neighborhood of $K$. We have for $\phi \in A$

$$
\begin{aligned}
\int_{\Omega} v & (x, t) \phi(x) d x \\
& =\sum_{k=0}^{\infty} \int\left(\int_{\Omega} E(x-y, t) \phi(x) d x\right) \Delta^{k} f_{k}(y) d y .
\end{aligned}
$$

By using Proposition 1.2 and (2.9) we can show that the right-hand side tends to

$$
v_{0}(\phi) \equiv \sum_{k=0}^{\infty} \Delta^{k} f_{k}(\phi)
$$

as $t \rightarrow 0_{+}$and $v_{0} \in A^{\prime}[K]$. This completes the proof of part (i).

The proof of parts (ii) and (iii) can be obtained similarly. We shall only prove the estimation $(2.3)_{s}$ for a given element $u \in \mathscr{E}_{K}^{\{s\}^{\prime}}, s>1$. We assume that for any $h>0$ and $\delta>0$, there is a constant $C=C(u, h, \delta)$ satisfying

$$
|u(\phi)| \leq C \sup _{\substack{x \in K_{\delta} \\ \alpha}} \frac{\left|D^{\alpha} \phi(x)\right|}{h^{|\alpha|} \alpha !^{s}}, \quad \phi \in \mathscr{E}^{\{s\}}\left(R^{n}\right) .
$$

Let $U(x, t)=u_{y}(E(x-y, t)),(x, t) \in R_{+}^{n+1}$. Then by using Proposition 1.1 we find

$$
\begin{aligned}
|U(x, t)| & \leq C_{h} \sup _{y \in K_{\delta}} \frac{\left|D_{y}^{\alpha} E(x-y, t)\right|}{h^{|\alpha|} \alpha !^{s}} \\
& \leq C C_{h} t^{-n / 2} \sup _{\alpha}\left[\frac{\left(C^{2} / h^{2} t\right)^{-|\alpha| /(2 s-1)}}{\alpha !}\right]^{(2 s-1) / 2} \sup _{y \in K_{\delta}} \exp \left[-(x-y)^{2} / 8 t\right] \\
& \leq C C_{h} t^{-n / 2} \exp \left[2 /(2 s-1)\left(C^{2} / h^{2} t\right)^{1 /(2 s-1)}\right] \exp \left[-\operatorname{dis}\left(x, K_{\delta}\right)^{2} / 8 t\right] .
\end{aligned}
$$


Taking $h>0$ sufficiently large so that

$$
(2 /(2 s-1))\left(C^{2} / h^{2}\right)^{1 /(2 s-1)}<\varepsilon
$$

we obtain $(2.3)_{s}$. The case where $u \in \mathscr{E}^{(s)^{\prime}}\left(R^{n}\right), s>1$, is treated by the similar way. We can easily find $(2.3)_{*}$ when $u \in \mathscr{E}^{\prime}\left(R^{n}\right)$. Q.E.D.

We can consider $A^{\prime}\left[K_{1}\right] \subset A^{\prime}\left[K_{2}\right]$ if $K_{1} \subset K_{2} \Subset R^{n}$ and we set $A^{\prime}\left(R^{n}\right)=$ $\bigcup_{K \Subset R^{n}} A^{\prime}[K]$.

Theorem 2.2 (cf. [3, Theorem 9.1.6]). If $u \in A^{\prime}\left(R^{n}\right)$ then there is a smallest compact set $K \subset R^{n}$ such that $u \in A^{\prime}[K]$; it is called the support of $u$. If $u \in \mathscr{E}^{\prime}\left(R^{n}\right)$ or $u \in \mathscr{E}^{\{s\}^{\prime}}\left(R^{n}\right)(s>1)$, then $u$ defines an element in $A^{\prime}\left(R^{n}\right)$ with the same support.

Proof. Let $u \in A^{\prime}[K]$ and let $K$ be the intersection of all compact sets $K^{\prime}$ such that $u \in A^{\prime}\left[K^{\prime}\right]$. By Theorem 2.1 a function $U(x, t)$ is uniquely defined and satisfies the heat equation in $R^{n+1} \backslash(K \times\{0\})$. Noting that $u=\lim _{t \rightarrow 0} U(\cdot, t)$, we see $u \in A^{\prime}[K]$. If $u \in \mathscr{E}^{\prime}\left(R^{n}\right)$ or $u \in \mathscr{E}^{\{s\}^{\prime}}\left(R^{n}\right)$ the function $U(x, t)$ is also uniquely defined and $U(\cdot, t) \rightarrow u$ in $\mathscr{E}^{\prime}\left(R^{n}\right)$ or in $\mathscr{E}^{\{s\}^{\prime}}\left(R^{n}\right)$ as $t \rightarrow 0_{+}$. So continuation of $U$ as a solution of heat equation is only possible outside of supp $u \times\{0\}$.

Theorem 2.3 (cf. [3, Theorem 9.1.8]). If $K_{1}, \ldots, K_{r}$ are compact subsets of $R^{n}$ and $u \in A^{\prime}\left[K_{1} \cup \cdots \cup K_{r}\right]$, then one can find $u_{j} \in A^{\prime}\left[K_{j}\right]$ so that $u=u_{1}+\cdots+u_{r}$. Proof. It is sufficient to prove the statement when $r=2$. Let $U(x, t)$ be the function defined by (2.1). The theorem will be proved if we can split $U$ into a sum $U=U_{1}+U_{2}$, where $U_{j}$ satisfies conditions (2.2) and (2.3) with respect to $K_{j}, j=1,2$. By virtue of condition (2.3) we can extend $U$ beyond the hyperplane $t=0$ as an ultradistribution $\widetilde{U} \in \mathscr{D}^{\{2\}^{\prime}}\left(R^{n+1}\right)$ such that

$$
\tilde{U}= \begin{cases}U(x, t), & t>0, \\ 0, & t<0 .\end{cases}
$$

Then $\widetilde{U}$ satisfies the heat equation outside of $\widetilde{K}_{1} \cup \widetilde{K}_{2}$ in the ordinary sense, where $\widetilde{K}_{j}=K_{j} \times\{0\}, j=1,2$. We take a function $\psi \in C^{\infty}\left(R^{n+1} \backslash\left(\widetilde{K}_{1} \cap \widetilde{K}_{2}\right)\right)$ constructed in [3, Corollary 1.4.11] such that $\psi=0$ for large $|x|+t$ and near $\widetilde{K}_{2} \backslash\left(\widetilde{K}_{1} \cap \widetilde{K}_{2}\right), \psi=1$ near $\widetilde{K}_{1} \backslash\left(\widetilde{K}_{1} \cap \widetilde{K}_{2}\right)$ and $\psi \in L^{\infty}\left(R^{n+1}\right)$. Here "near" means in the sense of the slowly varying metric defined in [3, Chapter 1]. We will split $\widetilde{U}$ as follows:

$$
\tilde{U}_{1}=\psi \tilde{U}-\tilde{V}, \quad \tilde{U}_{2}=(1-\psi) \tilde{U}+\tilde{V} .
$$

Here we define $\psi \widetilde{U} \in \mathscr{E}^{\{2\}^{\prime}}\left(R^{n+1}\right)$ such that $\psi \widetilde{U}=0$ near $\widetilde{K}_{2} \backslash\left(\widetilde{K}_{1} \cap \widetilde{K}_{2}\right)$ and $(1-\psi) \tilde{U}=0$ near $\tilde{K}_{1} \backslash\left(\tilde{K}_{1} \cap \widetilde{K}_{2}\right)$. We can write

$$
(\partial / \partial t-\Delta) \psi \widetilde{U}=\widetilde{F}+f
$$


where $\widetilde{F}$ and $f$ are in $\mathscr{E}^{\{2\}^{\prime}}\left(R^{n+1}\right)$ such that

$$
\widetilde{F}= \begin{cases}(\partial / \partial t-\Delta)(\psi U), & t>0, \\ 0, & t<0\end{cases}
$$

and $f \in \mathscr{E}^{\{2\}^{\prime}}\left(R^{n+1}\right)$, supp $f \subset \widetilde{K}_{1}$. Now we define

$$
\tilde{V}(x, t) \equiv E * \widetilde{F}(x, t) \in \mathscr{D}^{\{2\}^{\prime}}\left(R^{n+1}\right)
$$

and $V(x, t) \equiv \widetilde{V}(x, t)$ for $t>0$. Then we have $\widetilde{V} \in C^{\infty}\left(R^{n+1} \backslash \widetilde{K}_{1} \cap \widetilde{K}_{2}\right)$, $\operatorname{supp} \tilde{V} \subset \overline{R_{+}^{n+1}}$ and $V(\cdot, t) \rightarrow 0$ uniformly in $\left\{x ; \operatorname{dis}\left(x, K_{1} \cap K_{2}\right) \geq \delta\right\}$ for every $\delta>0$ as $t \rightarrow 0_{+}$. Here we used the pseudolocal property of $E *$ as a pseudodifferential operator in the class $S_{1 / 2,0,1}$ (cf. [10 and 11]). Since we have

$$
V(x, t)=\psi U-E * f(x, t), \quad t>0,
$$

we have for any $\varepsilon>0$

$$
V(x, t)=O\left(e^{\varepsilon / t}\right) \text { as } t \rightarrow 0_{+} .
$$

Thus we have the desired property that $U_{1}=\psi U-V$ and $U_{2}=(1-\psi) U+V$ for $t>0$. Q.E.D.

We will define the general hyperfunctions following [3].

Definition 2.1. Let $\Omega$ be a bounded open set in $R^{n}$. The space of hyperfunctions $B(\Omega)$ is defined by

$$
B(\Omega)=A^{\prime}[\bar{\Omega}] / A^{\prime}[\partial \Omega] .
$$

Definition 2.2. Let $u \in A^{\prime}[\bar{\Omega}]$. Then the support of the class $\dot{u}$ of $u$ in $B(\Omega)$ is defined by supp $\dot{u}=\Omega \cap \operatorname{supp} u$.

This definition is justified by the following fact: If $u, v \in A^{\prime}[\bar{\Omega}]$ and $u-$ $v \in A^{\prime}[\partial \Omega]$, then $\Omega \cap \operatorname{supp} u=\Omega \cap \operatorname{supp} v$ since supp $u \subset \operatorname{supp} v \cup \partial \Omega$ and $\operatorname{supp} v \subset \operatorname{supp} u \cup \partial \Omega$.

Definition 2.3. Let $\omega$ and $\Omega$ be open, bounded and $\omega \subset \Omega \subset R^{n}$. Let $u \in$ $A^{\prime}[\bar{\Omega}]$. Then the restriction to $\omega$ of the class $\dot{u}$ in $B(\Omega)$ is defined by the class $\dot{v}$ of $v \in A^{\prime}[\bar{\omega}]$ in $B(\omega)$, where $\omega \cap \operatorname{supp}(u-v)=\varnothing$.

We remark that for every $u \in A^{\prime}[\bar{\Omega}]$ we can find an element $v \in A^{\prime}[\bar{\omega}]$ such that $\omega \cap \operatorname{supp}(u-v)=\varnothing$ and the restriction of $u$ to $\omega$ is uniquely determined.

Now we state a localization theorem given in [3]. We shall base its proof on Theorem 2.1 and Theorem 2.3.

Theorem 2.4 (cf. [3, Theorem 9.2.2]). Let $\Omega_{j}$ be open sets in $R^{n}$ with bounded union $\Omega$. If $u_{j} \in B\left(\Omega_{j}\right)$ and for all $i, j$ we have $u_{i}=u_{j}$ in $\Omega_{i} \cap \Omega_{j}$ (that is, the restrictions are equal) then there is a unique $u \in B(\Omega)$ such that the restriction of $u$ to $\Omega_{j}$ is equal to $u_{j}$ for every $j$.

As pointed out at the end of $[3, \S 9.2]$, it follows from this theorem that we have injections $\mathscr{D}^{\prime}(\Omega) \rightarrow \mathscr{D}^{\{s\}^{\prime}}(\Omega) \rightarrow B(\Omega) \quad(1<s<\infty)$. Let us also note that 
the elements with compact support in $B(\Omega)$ can be identified with the elements in $A^{\prime}$ having support in $\Omega$. In fact, let $u \in A^{\prime}[\bar{\Omega}]$ and assume that the class $u$ has compact support $K \subset \Omega$. Then supp $u \subset K \cup \partial \Omega$ so Theorem 2.2 gives a decomposition

$$
u=u_{1}+u_{2}, \quad u_{1} \in A^{\prime}[K], \quad u_{2} \in A^{\prime}[\partial \Omega]
$$

which is unique since $K$ and $\partial \Omega$ are disjoint. This means that $\dot{u}=\dot{u}_{1}$ for a unique $u_{1} \in A^{\prime}[K]$.

Proof of Theorem 2.4. The uniqueness is clear for $v$ has the same property as $u$, then the support of $u-v$ is empty so $u-v=0$. To prove the existence we begin with the case of two open sets $\Omega_{1}$ and $\Omega_{2}$. Choose $\bar{u}_{j} \in A^{\prime}\left[\bar{\Omega}_{j}\right]$ defining $u_{j}$ for $j=1,2$. The support of $u_{1}-u_{2}$ is contained in

$$
\left(\bar{\Omega}_{1} \cup \bar{\Omega}_{2}\right) \backslash\left(\Omega_{1} \cap \Omega_{2}\right) \subset\left(\complement \Omega_{1} \cap \bar{\Omega}_{2}\right) \cup\left(\bar{\Omega}_{1} \cap \complement \Omega_{2}\right),
$$

so Theorem 2.2 gives a decomposition

$$
\bar{u}_{1}-\bar{u}_{2}=\bar{v}_{1}-\bar{v}_{2}, \quad \bar{v}_{1} \in A^{\prime}\left[\complement \Omega_{1} \cap \bar{\Omega}_{2}\right], \quad \bar{v}_{2} \in A^{\prime}\left[\bar{\Omega}_{1} \cap \complement \Omega_{2}\right] .
$$

Now $\bar{u}=\bar{u}_{1}-\bar{v}_{1}=\bar{u}_{2}-\bar{v}_{2}$ defines an element in $B\left(\Omega_{1} \cup \Omega_{2}\right)$ which restricts to $u_{j}$ in $\Omega_{j}$ for $j=1,2$.

Next we assume that we have countably many sets $\Omega_{j}, j=1,2, \ldots$ Repeated use of the special case just proved gives a sequence $w_{j}$ in $B\left(\Omega_{1} \cup \cdots \cup \Omega_{j}\right)$ with restriction $u_{i}$ to $\Omega_{i}$ for $i \leq j$. Let $\bar{w}_{j} \in A^{\prime}\left[\overline{\Omega_{1} \cdots \Omega_{j}}\right]$ be in the class of $w_{j}, j=1,2, \ldots$. The proof will be complete if we shall show that there is an element $\bar{w} \in A^{\prime}[\bar{\Omega}]$ such that $\operatorname{supp}\left(\bar{w}-\bar{w}_{j}\right)$ in $\bar{\Omega} \backslash\left(\Omega_{1} \cup \cdots \cup \Omega_{j}\right)$ for every $j$. As is easily seen, we may suppose that $\Omega_{j}=\{x ; x \in \Omega, \operatorname{dis}(x, \partial \Omega)>1 / j\}, j=$ $1,2, \ldots$. Let $W_{j}(x, t)$ be the defining function of $\bar{w}_{j}$;

$$
W_{j}(x, t)=\int E(x-y, t) \bar{w}_{j}(y) d y, \quad t>0,
$$

where the integration is taken in the distribution sense. We shall show that there are defining functions $V_{j}(x, t), j=1,2, \ldots$, with the following properties:

$$
(\partial / \partial t-\Delta) V_{j}(x, t)=0 \quad \text { in } R_{+}^{n+1} ;
$$

for any $\varepsilon>0$,

$$
\left|V_{j}(x, t)\right| \leq C_{j, \varepsilon} e^{\varepsilon / t} \text { in } R_{+}^{n+1}
$$

for every $\delta>0$,

$$
V_{j}(\cdot, t) \rightarrow 0 \text { as } t \rightarrow 0_{+}
$$

uniformly in $\left\{x \in R^{n} ; \operatorname{dis}(x, \partial \Omega) \geq \delta\right\}$; there exists a positive constant $C$ such that

$$
\left|W_{j+1}-W_{j}-V_{j}\right| \leq C 2^{-j} \exp \left[\varepsilon_{j} / t\right] \text { in } R_{+}^{n+1}
$$


where $\varepsilon_{j} \rightarrow 0$ as $j \rightarrow \infty$; for every $\delta>0$, there is a number $j_{\delta}$ such that

$$
\left|W_{j+1}(\cdot, t)-W_{j}(\cdot, t)-V_{j}(\cdot, t)\right| \leq C_{\delta} 2^{-j} \exp \left[-c_{\delta} / t\right]
$$

in $\left\{x \in R^{n} ; \operatorname{dis}(x, \partial \Omega) \geq \delta\right\}$ for all $j \geq j_{\delta}$, where $C_{\delta}$ and $c_{\delta}$ can be taken independent of $j$. If these are proved then it follows from (2.24) that the limit

$$
\begin{aligned}
W(x, t) & =\lim \left(W_{j}-V_{1}-\cdots-V_{j-1}\right) \\
& =W_{j}-V_{1}-\cdots-V_{j-1}+\sum_{j}^{\infty}\left(W_{k+1}-W_{k}-V_{k}\right)
\end{aligned}
$$

exists for $(x, t) \in R_{+}^{n+1}$ and $W(x, t)$ defines $\bar{w}=\lim _{t \rightarrow 0_{+}} W(\cdot, t)$ which is the desired element in $A^{\prime}[\bar{\Omega}]$.

It remains to show the existence of such defining functions $V_{j}(x, t), j=$ $1,2, \ldots$. For each $j$ the compact region $\overline{\boldsymbol{\Omega}}_{j} \backslash \mathbf{\Omega}_{j+1} \quad(\subset \Omega)$ can be covered by the finite number of balls with radius $2 / j$ and center on $\partial \Omega$, say $B_{j k}=\{x ; \mid x-$ $\left.x_{j k} \mid<2 / j\right\}, x_{j k} \in \partial \Omega, k=1, \ldots, k_{j}$. Since $\operatorname{supp}\left(\bar{w}_{j+1}-\bar{w}_{j}\right) \subset \bar{\Omega}_{j} \backslash \Omega_{j+1}$, we can split $\bar{w}_{j+1}-\bar{w}_{j}$ as follows:

$$
\bar{w}_{j+1}-\bar{w}_{j}=\sum_{k=1}^{k_{j}} g_{j k}, \quad \operatorname{supp} g_{j k} \subset \bar{B}_{j k}, k=1, \ldots, k_{j} .
$$

Thus we have by $(2.20)$

$$
W_{j+1}(x, t)-W_{j}(x, t)=\sum_{k=1}^{k_{j}} g_{j k}(E(x-\cdot, t)), \quad t>0 .
$$

By taking the Taylor expansion of $E$ at $x-x_{j k}$ we have

$$
\int E(x-y, t) g_{j k}(y) d y=\sum_{\alpha} \frac{1}{\alpha !} \partial_{x}^{\alpha} E\left(x-x_{j k}, t\right) \int\left(x_{j k}-y\right)^{\alpha} g_{j k}(y) d y,
$$

where the integration is taken in the sense of distributions. By Theorem 1.1 we may suppose that for any $h>0$ there is a constant $C=C(h, j)$ such that

$$
\left|g_{j k}(\phi)\right| \leq C \sup _{\substack{x \in \bar{B}_{j k} \\ \alpha}} \frac{\left|D^{\alpha} \phi(x)\right|}{h^{|\alpha|} \alpha !}, \quad \phi \in A, k=1, \ldots, k_{j} .
$$

We set

$$
V_{j k}=\sum_{|\alpha| \leq N} \frac{1}{\alpha !} \partial_{x}^{\alpha} E\left(x-x_{j k}, t\right) \int\left(x_{j k}-y\right)^{\alpha} g_{j k}(y) d y,
$$


where the number $N$ will be determined later. Then we see by using Proposition 1.1 that

$$
\begin{aligned}
& \left|\int E(x-y, t) g_{j k}(y) d y-V_{j k}(x, t)\right| \\
& \quad=\left|\sum_{|\alpha| \geq N+1} \frac{1}{\alpha !} \partial_{x}^{\alpha} E\left(x-x_{j k}, t\right) \int\left(x_{j k}-y\right)^{\alpha} g_{j k}(y) d y\right| \\
& \quad \leq C \sum_{|\alpha| \geq N+1} C_{n}^{|\alpha|} t^{-(n+|\alpha|) / 2} \alpha !^{-1 / 2} \exp \left[-\left(x-x_{j k}\right)^{2} / 8 t\right](2 / j)^{|\alpha|} \\
& \quad \leq C t^{-n / 2} \exp \left[\frac{\varepsilon}{t}\right] \exp \left[-\left(x-x_{j k}\right)^{2} / 8 t\right] \sum_{|\alpha| \geq N+1}\left(\frac{2 C_{n}}{j \sqrt{\varepsilon}}\right)^{|\alpha|},
\end{aligned}
$$

where $\varepsilon>0$ and $C_{n}$ depends only on the dimension $n$ of $R^{n}$. Taking $\varepsilon=$ $\varepsilon_{j}^{\prime}=\left(4 C_{n} / j\right)^{2}$ we have

$$
\begin{aligned}
& \left|\int E(x-y, t) g_{j k}(y) d y-V_{j k}\right| \\
& \leq C_{j} t^{-n / 2} \exp \left[\frac{\varepsilon_{j}^{\prime}}{t}\right] \exp \left[-\left(x-x_{j k}\right)^{2} / 8 t\right] \sum_{|\alpha| \geq N+1}\left(\frac{1}{2}\right)^{|\alpha|} .
\end{aligned}
$$

Thus by taking $N$ sufficiently large, we have

$$
\left|W_{j+1}(x, t)-W_{j}(x, t)-\sum_{k=1}^{k_{j}} V_{j k}\right| \leq 2^{-j} t^{-n / 2} \exp \left[\varepsilon_{j}^{\prime} / t\right], \quad \varepsilon_{j}^{\prime}=\left(4 C_{n} / j\right)^{2} .
$$

If we set

$$
V_{j}(x, t)=\sum_{k=1}^{k_{j}} V_{j k}(x, t), \quad j=1,2, \ldots,
$$

assertions (2.21)-(2.24) are proved and we can easily verify (2.25) similarly as above. Q.E.D.

We shall finish this section by giving local existence theorem as an application of Theorem 2.1. Let $P(D)$ be a differential operator with constant coefficients and let $u \in A^{\prime}[K], K \Subset R^{n}$. Then $P(D) u \in A^{\prime}[K]$ is uniquely defined by the usual way:

$$
P(D) u(\phi) \equiv u(P(-D) \phi), \quad \phi \in A[K] .
$$

Let $U(x, t)$ be the defining function of $u$. Then $P(D) u$ is also given as a limit $P(D) u=\lim _{t \rightarrow 0_{+}} P(D) U(\cdot, t)$ in the sense of (2.4).

Theorem 2.5. Let $\Omega$ be a bounded open subset of $R^{n}$. Let $P(D)$ be a differential operator with constant coefficients and consider the equation

$$
P(D) u=f, \quad f \in B(\Omega) .
$$


For any $f \in B(\Omega)$ we can find a solution $u \in B(\Omega)$ to the equation (2.27).

Proof. Let $f \in A^{\prime}[\bar{\Omega}]$ and let $F(x, t)$ be the defining function of $f$ given by

$$
F(x, t)=f(E(x-\cdot, t)), \quad(x, t) \in R_{+}^{n+1} .
$$

We know that there always exists a fundamental solution $G \in \mathscr{D}^{\prime}\left(R^{n}\right)$ of $P(D)$ such that $P(D) G=\delta$ in $R^{n}$ (cf. [2]). Let $\chi(x) \in C_{0}^{\infty}\left(R^{n}\right)$ such that $\chi(x)=1$ in a neighborhood of $\bar{\Omega}$. We consider a defining function

$$
U(x, t) \equiv(\chi G) \underset{(x)}{*} F(x, t), \quad(x, t) \in R_{+}^{n+1} .
$$

We can easily verify that $U$ satisfies conditions (2.2) and (2.3) with $K$ replaced by some compact set $K_{1} \ni \bar{\Omega}$. Let

$$
u_{0}=\lim _{t \rightarrow 0_{+}} U(\cdot, t) \in A^{\prime}\left[K_{1}\right] .
$$

By Theorem 2.3, $u_{0}$ can be written as

$$
u_{0}=u_{1}+u_{2}, \quad u_{1} \in A^{\prime}[\bar{\Omega}], \quad u_{2} \in A^{\prime}\left[K_{1} \backslash \Omega\right] .
$$

Then the class of $u_{1}$ in $B(\Omega)$ gives a solution of equation (2.27). Q.E.D.

\section{LOCAL REGULARITY}

Let $u \in A^{\prime}\left(R^{n}\right)$. Then $U(x, t)=u_{y}(E(x-y, t))$ defined in $(2.1)$ is a $C^{\infty}$. function in the half space $R_{+}^{n+1}$. Furthermore $U(\cdot, t)$ is extensible as entire analytic function in $C^{n}$ for each $t>0$. We shall consider the regularity property of $u \in A^{\prime}\left(R^{n}\right)$ relating to the asymptotic property of $U(z, t)$ as $t \rightarrow$ $0, z=x+i \xi \in C^{n}$.

Theorem 3.1. Let $u \in A^{\prime}\left(R^{n}\right)$. Then

(i) $u$ is analytic in a neighborhood of $x_{0} \in R^{n}$ if and only if for any $\zeta_{0} \in$ $R^{n} \backslash\{0\}$, there is a neighborhood $V\left(x_{0}, \xi_{0}\right) \subset T^{*}\left(R^{n}\right) \backslash 0$ and there are positive constants $C$ and $c$ such that

$$
|U(x+i \xi, t)| \leq C \exp \left[\left(\xi^{2} / 4 t\right)-(c / t)\right], \quad t>0,(x, \xi) \in V\left(x_{0}, \xi_{0}\right) .
$$

(ii) $u$ is in $\mathscr{E}^{\{s\}}(s>1)$ in a neighborhood of $x_{0} \in R^{n}$ if and only if for any $\xi_{0} \in R^{n} \backslash 0$

$$
|U(x+i \xi, t)| \leq C \exp \left[\left(\xi^{2} / 4 t\right)-(c / t)^{1 / s}\right], \quad t>0,(x, \xi) \in V\left(x_{0}, \xi_{0}\right),
$$

where $V\left(x_{0}, \xi_{0}\right), C$ and $c$ are taken as in (3.1).

(iii) $u$ is a $C^{\infty}$-function in a neighborhood of $x_{0} \in R^{n}$ if and only if for any $\xi_{0} \in R^{n} \backslash 0$ there is a neighborhood $V\left(x_{0}, \xi_{0}\right) \subset T^{*}\left(R^{n}\right) \backslash 0$ such that

$$
|U(x+i \xi, t)| \leq C_{N} \exp \left[\xi^{2} / 4 t\right] t^{N}, \quad t>0,(x, t) \in V\left(x_{0}, \xi_{0}\right),
$$


where $N$ is an arbitrary positive integer and $C_{N}$ is a constant depending on $N$. Proof. Let $u \in A^{\prime}\left(R^{n}\right)$. Then we have

$$
\begin{aligned}
U(x+i \xi, t)= & (4 \pi t)^{-n / 2} \exp \left[\xi^{2} / 4 t-i\langle x, \xi\rangle / 2 t\right] \\
& \cdot u_{y}\left(\exp \left[-(x-y)^{2} / 4 t+i\langle y, \xi\rangle / 2 t\right]\right), \\
& \quad(x+i \xi) \in \mathbf{C}^{n}, t>0 .
\end{aligned}
$$

The essential part which we have to treat is the following:

$$
\tilde{u}(x, t, \xi) \equiv(4 \pi t)^{-n / 2} u_{y}\left(\exp \left[-(x-y)^{2} / 4 t+i\langle y, \xi\rangle / 2 t\right]\right), \quad t>0 .
$$

Thus we see $(4 \pi t)^{n / 2} \tilde{u}$ is nothing but the generalized Fourier transformation of Bros-Iagolnitzer with parameter $\lambda=1 / 2 t$.

Now we will first prove the part (i). Let $u \in A^{\prime}\left(R^{n}\right)$ and let $u$ be real analytic in a neighborhood of $x_{0} \in R^{n}$, say in $\omega_{\delta}=\left\{x ;\left|x-x_{0}\right|<\delta\right\}$ for some $\delta>0$. Then by Theorem 2.3 we can divide $u$ so that

$$
u=u_{1}+u_{2}, \quad \operatorname{supp} u_{1} \subset \bar{\omega}_{\delta}, \quad \operatorname{supp} u_{2} \subset \complement \omega_{\delta} .
$$

Obviously $u_{1} \in \mathscr{E}^{\prime}\left(R^{n}\right)$ and $u_{1}$ is analytic in $\omega_{\delta}$. We divide $U(x, t)$ as follows:

$$
\begin{gathered}
U(x, t)=U_{1}(x, t)+U_{2}(x, t), \\
U_{j}(x, t)=u_{j y}(E(x-y, t)), \quad j=1,2 .
\end{gathered}
$$

We have

$$
\tilde{u}_{1}(x, t, \xi)=(4 \pi t)^{-n / 2} u_{1 y}\left(\exp \left[-(x-y)^{2} / 4 t+i\langle y, \xi\rangle / 2 t\right]\right), \quad t>0 .
$$

By deformation of the integration contour we can see that analyticity of $u_{1}$ in $\omega_{\delta}$ yields the estimation of the form

$$
\left|\tilde{u}_{1}(x, t, \xi)\right| \leq C \exp [-c / t], \quad(x, \xi) \in V\left(x_{0}, \xi_{0}\right), t>0,
$$

where $V\left(x_{0}, \xi_{0}\right)=\omega_{\delta / 2} \times\left\{\xi ;\left|\xi-\xi_{0}\right|<\delta^{\prime}\right\}$ and $\delta^{\prime}$ is an arbitrary positive number. As for $U_{2}$ we have to estimate

$$
\tilde{u}_{2}(x, t, \xi)=(4 \pi t)^{-n / 2} u_{2 y}\left(\exp \left[-(x-y)^{2} / 4 t+i\langle y, \xi\rangle / 2 t\right]\right), \quad t>0 .
$$

We shall apply the estimation $(1.11)^{\prime}$. Let $\Omega_{\varepsilon}$ be a complex neighborhood of $\operatorname{supp} u_{2}$ such that

$$
\Omega_{\varepsilon}=\left\{y+i \eta ; \operatorname{dis}\left(y, \operatorname{supp} u_{2}\right)<\varepsilon,|\eta|<\varepsilon\right\}, \quad \varepsilon>0 .
$$

We have by $(1.11)^{\prime}$

$$
\left|\tilde{u}_{2}(x, t, \xi)\right| \leq C_{\varepsilon}(4 \pi t)^{-n / 2} \sup _{\Omega_{\varepsilon}}\left|\exp \left[-(x-(y+i \eta))^{2} / 4 t+i\langle y+i \eta, \xi\rangle / 2 t\right]\right| .
$$


Let $x \in \omega_{\delta / 2}$. Then we have

$$
\begin{aligned}
\operatorname{Re}\{ & \left.-(x-(y+i \eta))^{2} / 4 t+i\langle y+i \eta, \xi\rangle / 2 t\right\} \\
& =-\left[(x-y)^{2}-\eta^{2}\right] / 4 t-\langle\xi, \eta\rangle / 2 t \\
& \leq-\left((\delta / 2-\varepsilon)^{2}-\varepsilon^{2}\right) / 4 t+\varepsilon|\xi| / 2 t, \quad 0<\varepsilon<\delta / 2 .
\end{aligned}
$$

Thus by taking $\varepsilon$ sufficiently small we find the estimation of the form

$$
\left|\tilde{u}_{2}\right| \leq C e^{-c / t}, \quad(x, \xi) \in V\left(x_{0}, \xi_{0}\right), \quad t>0,
$$

where $V\left(x_{0}, \xi_{0}\right)=\omega_{\delta / 2} \times\left\{\xi ;\left|\xi-\xi_{0}\right|<\delta^{\prime}\right\}$. The constants $C$ and $c$ may depend on $\delta, \delta^{\prime}$ and $\varepsilon$. These considerations imply that there are another couple of constants $C$ and $c$ such that

$$
|\tilde{u}(x, t, \xi)| \leq C e^{-c / t}, \quad x \in \omega_{\delta}, \xi \in S^{n-1}, t>0 .
$$

We put $\xi^{\prime}=\xi / 2 t$ in $(3.1)^{\prime}$ and rewrite $\xi^{\prime}$ as $\xi$ to get

$$
\begin{aligned}
& (|\xi| / 2)^{n / 2}\left|u_{y}\left(\exp \left[-|\xi|(x-y)^{2} / 2+i\langle y, \xi\rangle\right]\right)\right| \leq C \exp [-c|\xi|], \\
& \quad x \in \omega_{\delta}, \xi \in R^{n} \backslash\{0\} .
\end{aligned}
$$

We shall now follow the reasoning given in the proof of Lemma 2.2 in [14]. Since

$$
(|\xi| / 2 \pi)^{n / 2} \int_{R^{n}} \exp \left[-|\xi|(\beta-y)^{2} / 2\right] d \beta=1
$$

we have symbolically

$$
(2 \pi)^{-n} \iint(|\xi| / 2 \pi)^{n / 2} \exp \left[-|\xi|(\beta-y)^{2} / 2-i\langle x-y, \xi\rangle\right] d \beta d \xi=\delta(x-y)
$$

and then we have symbolically

$$
\text { (3.7) } u(x)=(2 \pi)^{-n} \iint(|\xi| / 2 \pi)^{n / 2} u_{y}\left(\exp \left[-|\xi|(\beta-y)^{2} / 2-i\langle x-y, \xi\rangle\right]\right) d \beta d \xi \text {. }
$$

We shall justify this for $u \in A^{\prime}\left(R^{n}\right)$ in the oscillatory sense and by applying (3.6) to the right-hand side of (3.7) we shall obtain the analyticity of $u$ near $x_{0}$. We set

$$
\begin{array}{r}
I_{\varepsilon}(x, y)=(2 \pi)^{-n} \iint(|\xi| / 2 \pi)^{n / 2} \exp \left[-|\xi|(\beta-y)^{2} / 2-i\langle x-y, \xi\rangle-\varepsilon|\xi|^{2}\right] d \beta d \xi, \\
\varepsilon>0,
\end{array}
$$

where the convergence factor $\exp \left[-\varepsilon|\xi|^{2}\right]$ guarantees absolute convergence. We want to prove that

$$
I_{\varepsilon}(x, y) \rightarrow \delta(x-y) \quad \text { as } \varepsilon \rightarrow 0 \text { in } A^{\prime}[K], \quad K \supset \operatorname{supp} u .
$$

Namely, for any $\phi \in A[K]$ (or $A$ )

$$
\left\langle u_{y}\left(I_{\varepsilon}(x, y)\right), \chi \phi\right\rangle \rightarrow\langle u, \phi\rangle \quad \text { as } \varepsilon \rightarrow 0 \text {, }
$$


where $\chi$ is a definition function of a bounded neighborhood of $K$ (cf. (2.4)). We have

$$
\begin{aligned}
& \left\langle u_{y}\left(I_{\varepsilon}(x, y)\right), \chi \phi\right\rangle \\
& \quad=(2 \pi)^{-n} \iiint(|\xi| / 2 \pi)^{n / 2} u_{y}\left(\exp \left[-|\xi|(\beta-y)^{2} / 2+i\langle x-y, \xi\rangle\right]\right) \\
& \cdot \exp \left[-\varepsilon \xi^{2}\right](\chi \phi)(x) d \beta d \xi d x \\
& \quad=u_{y}\left((2 \pi)^{-n} \int \exp \left[i\langle y, \xi\rangle-\varepsilon \xi^{2}\right] \widehat{\chi \phi}(\xi) d \xi\right), \quad \varepsilon>0 .
\end{aligned}
$$

Since

$$
(2 \pi)^{-n} \int \exp \left[i\langle y, \xi\rangle-\varepsilon \xi^{2}\right] d \xi=(4 \pi \varepsilon)^{-n / 2} \exp \left[-y^{2} / 4 \varepsilon\right], \quad \varepsilon>0,
$$

we have

$$
(2 \pi)^{-n} \int \exp \left[i\langle y, \xi\rangle-\varepsilon \xi^{2}\right] \widehat{\chi \phi}(\xi) d \xi=\int E(x-y, \varepsilon)(\chi \phi)(y) d y
$$

which tends to $\phi(x)$ in $A[K]$ as $\varepsilon \rightarrow 0_{+}$as a result of Proposition 1.2. Thus equation (3.7) is justified in the oscillatory sense for $u \in A^{\prime}\left(R^{n}\right)$ and we have with an entire function $g$ :

$$
\begin{array}{r}
\delta(x-y)=(2 \pi)^{-n} \iint_{|\xi| \geq 1} \exp \left[i\langle x-y, \xi\rangle-|\xi|(\beta-y)^{2} / 2\right] \\
\cdot(|\xi| / 2 \pi)^{n / 2} d \xi d \beta+g(x, y) .
\end{array}
$$

It follows that the first term in the right-hand side is analytic for $x \neq y$. In particular, in the region $\left|x-x_{0}\right|<\varepsilon,\left|y-x_{0}\right|>\varepsilon$, we conclude that the function

$$
f(x, y)=\iint_{\substack{\left|\beta-x_{0}\right|<2 \varepsilon \\|\xi|>1}} \exp \left[i\langle x-y, \xi\rangle-|\xi|(\beta-y)^{2} / 2\right]|\xi|^{n / 2} d \xi d \beta
$$

is analytic. On the other hand the phase has a strictly positive imaginary part when $\left|x-x_{0}\right|<\varepsilon$ and $\left|y-x_{0}\right|<2 \varepsilon$ so $f$ is analytic in that region also and hence in $\left\{(x, y) \in R^{2 n} ;\left|x-x_{0}\right|<\varepsilon\right\}$. Thus for $u \in A^{\prime}\left(R^{n}\right)$, we have for $\left|x-x_{0}\right|<\varepsilon$ :

$$
\begin{array}{r}
u(x)=(2 \pi)^{-n} \iint_{\substack{\left|\beta-x_{0}\right| \leq 2 \varepsilon \\
|\xi| \geq 1}} u_{y}\left(\exp \left[-i\langle x-y, \xi\rangle-|\xi|(\beta-y)^{2} / 2\right]\right) \\
\cdot(|\xi| / 2 \pi)^{n / 2} d \xi d \beta+w_{\varepsilon}(x),
\end{array}
$$

where $w_{\varepsilon}(x)$ is analytic. Choosing $\varepsilon<\delta$ we conclude that $u$ is analytic for $\left|x-x_{0}\right|<\varepsilon$ by virtue of (3.6). This completes the proof of part (i) of Theorem 3.1.

Proof of (ii). Let $u \in A^{\prime}\left(R^{n}\right)$ and assume that $u$ is in $\mathscr{E}^{\{s\}}(s>1)$ in a neighborhood of $x_{0} \in R^{n}$. Then we can divide $u$ so that $u=u_{1}+u_{2}$, where 
$u_{1} \in \mathscr{D}^{\{s\}}\left(\omega_{\delta}\right)$ and $\operatorname{supp} u_{2} \subset \complement \omega_{\delta_{1}}, \omega_{\delta}=\left\{x ;\left|x-x_{0}\right|<\delta\right\}, \delta>0,0<\delta_{1}<\delta$. As in the proof of (i), we have to estimate the following:

$$
\begin{aligned}
\tilde{u}_{j}(x, t, \xi)=(4 \pi t)^{-n / 2} u_{j y}\left(\exp \left[-(x-y)^{2} / 4 t+i\langle y, \xi\rangle / 2 t\right]\right) & \\
t & >0, j=1,2 .
\end{aligned}
$$

We first treat

$$
\tilde{u}_{1}(x, t, \xi)=(4 \pi t)^{-n / 2} \int u_{1}(y) \exp \left[-(x-y)^{2} / 4 t+i\langle y, \xi\rangle / 2 t\right] d y,
$$

where $u_{1}(y) \in \mathscr{D}^{\{s\}}\left(\omega_{\delta}\right), s>1$. Then there are positive constants $C_{0}$ and $C_{1}$ such that

$$
\sup \left|D^{\alpha} u_{1}(y)\right| \leq C_{0} C_{1}^{|\alpha|} \alpha !^{s}, \quad \alpha \in Z_{+}^{n} .
$$

By Proposition 1.1, taking $C_{1}$ sufficiently large if necessary we have

$$
\begin{aligned}
\mid D_{y}^{\alpha}\{ & \left.u_{1}(y) \exp \left[-(x-y)^{2} / 4 t\right]\right\} \mid \\
& \leq C_{0} C_{1}^{|\alpha|} \sum_{\beta \leq \alpha}\left(\begin{array}{c}
\alpha \\
\beta
\end{array}\right)(\alpha-\beta) !^{s} \beta !^{1 / 2} t^{-|\beta| / 2} \exp \left[-c(x-y)^{2} / t\right] \\
& \leq C_{0} C_{1}^{|\alpha|} \alpha !^{s} \sum_{\beta \leq \alpha}\left(\begin{array}{c}
\alpha \\
\beta
\end{array}\right)^{(1-s)} \beta !^{(1-2 s) / 2} t^{-|\beta| / 2} \\
& \leq C_{0}^{\prime} C_{1}^{|\alpha|} \alpha !^{s} \exp \left[c^{\prime} t^{-1 /(2 s-1)}\right]
\end{aligned}
$$

with another couple of constants $C_{0}^{\prime}$ and $c^{\prime}$. Here we have used the fact

$$
\sup _{\alpha} \sum_{\beta \leq \alpha}\left(\begin{array}{l}
\alpha \\
\beta
\end{array}\right)^{(1-s)}<\infty, \quad(s>1) .
$$

By making use of the above estimation we obtain the inequality of the form

$$
\left|\tilde{u}_{1}(x, t, \xi)\right| \leq A \exp \left[-a(|\xi| / t)^{1 / s}+c^{\prime}(1 / t)^{1 /(2 s-1)}\right]
$$

with positive constants $A$ and $a$. This yields the estimation of the form (3.13)

$$
\left|\tilde{u}_{1}(x, t, \xi)\right| \leq C \exp \left[-c(1 / t)^{1 / s}\right], \quad t>0,(x, \xi) \in V\left(x_{0}, \xi_{0}\right) \Subset T^{*}\left(R^{n}\right) \backslash 0 .
$$

As for $\tilde{u}_{2}$, we can also use $(1.11)^{\prime}$ as in the proof of (i) to get the estimation of the form

$$
\left|\tilde{u}_{2}\right| \leq C \exp [-c / t],(x, \xi) \in V\left(x_{0}, \xi_{0}\right), \quad t>0,
$$

where $V\left(x_{0}, \xi_{0}\right)$ is a sufficiently small neighborhood of $\left(x_{0}, \xi_{0}\right)$.

Next we assume (3.2) for $u \in A^{\prime}\left(R^{n}\right)$ and we shall show that $u$ is in $\mathscr{E}^{\{s\}}$ near $x_{0}$. Condition (3.2) implies that there are positive constants $C, c$ and $\delta$ such that

$$
|\tilde{u}(x, t, \xi)| \leq C \exp \left[-c(1 / t)^{1 / s}\right], \quad x \in \omega_{\delta}, \xi \in S^{n-1}, t>0 .
$$


This yields

$$
\begin{array}{r}
(|\xi| / 2 \pi)^{n / 2}\left[u_{y}\left(\exp \left[-|\xi|(x-y)^{2} / 2-i\langle y, \xi\rangle\right]\right) \mid \leq C \exp \left[-c|\xi|^{1 / s}\right],\right. \\
x \in \omega_{\delta}, \xi \in R^{n} \backslash\{0\} \text { (cf. (3.6)). }
\end{array}
$$

We have already equation (3.9). As in the proof of part (i) we have for $\left|x-x_{0}\right|$ $<\varepsilon$

$$
\begin{array}{r}
u(x)=(2 \pi)^{-n} \iint_{\substack{\left|\beta-x_{0}\right| \leq 2 \varepsilon \\
|\xi| \geq 1}} u_{y}\left(\exp \left[i\langle x-y, \xi\rangle-|\xi|(\beta-y)^{2} / 2\right]\right) \\
\cdot(|\xi| / 2 \pi)^{n / 2} d \xi d \beta+w_{\varepsilon}(x)
\end{array}
$$

where $w_{\varepsilon}(x)$ is analytic. Choosing $\varepsilon<\delta$ we conclude that $u$ is in $\mathscr{E}^{\{s\}}$ for $\left|x-x_{0}\right|<\varepsilon$ by using (3.15). This completes the proof of part (ii) of Theorem 3.1 .

Proof of (iii). Let $u \in A^{\prime}\left(R^{n}\right)$ and assume that $u$ is in a neighborhood of $x_{0} \in R^{n}$. Then we divide $u$ so that $u=u_{1}+u_{2}$, where $u_{1} \in \mathscr{D}\left(\omega_{\delta}\right)$ and $\operatorname{supp} u_{2} \subset \complement \omega_{\delta^{\prime}}, \omega_{\delta}=\left\{x ;\left|x-x_{0}\right|<\delta\right\}, 0<\delta^{\prime}<\delta$. As we have seen in the proof of parts (i) and (ii) we have only to estimate

$$
\left.\tilde{u}_{1}(x, t, \xi)=(4 \pi t)^{-n / 2} \int u_{1}(y) \exp \left[-(x-y)^{2} / 4 t+i\langle y, \xi\rangle / 2 t\right]\right) d y, \quad t>0 .
$$

We have obviously the estimate

$$
\left|D_{y}^{\alpha}\left\{u_{1}(y) \exp \left[-(x-y)^{2} / 4 t\right]\right\}\right| \leq C_{\alpha} t^{-|\alpha| / 2}
$$

with constants $C_{\alpha}$ depending on $\alpha \in Z_{+}^{n}$. On the other hand

$$
\begin{gathered}
(4 \pi t)^{-n / 2} \int D_{y}^{\alpha}\left\{u_{1}(y) \exp \left[-(x-y)^{2} / 4 t\right]\right\} \exp [i\langle y, \xi\rangle / 2 t] d y \\
=(-\xi / 2 t)^{\alpha} \tilde{u}_{1}(x, t, \xi),
\end{gathered}
$$

from where we have for each integer $N \geq 0$

$$
\left|\tilde{u}_{1}(x, t, \xi)\right| \leq C_{N}(4 \pi t)^{-n / 2} t^{N / 2}|\xi|^{-N}, \quad t>0, \xi \in R^{n} \backslash\{0\} .
$$

Thus we get the estimation of the form (3.3).

Conversely we assume (3.3) for $u \in A^{\prime}\left(R^{n}\right)$ and we shall show that $u$ is in $\mathscr{E}$ near $x_{0}$. Condition (3.3) implies that there are positive constants $C_{N}$ and $\delta$ such that

$$
|\tilde{u}(x, t, \xi)| \leq C_{N} t^{N}, \quad x \in \omega_{\delta}, \xi \in S^{n-1}, t>0 .
$$

This yields the estimate of the form

$$
\begin{array}{r}
(|\xi| / 2 \pi)^{n / 2}\left|u_{y}\left(\exp \left[-|\xi|(x-y)^{2} / 2+i\langle y, \xi\rangle\right]\right)\right| \leq C_{N}|\xi|^{-N} \\
N \geq 0, x \in \omega_{\delta}, \xi \in R^{n} \backslash\{0\}
\end{array}
$$


As in the proof of part (i) we have equation (3.6) from which we find that $u$ is a $C^{\infty}$-function for $\left|x-x_{0}\right|<\varepsilon$ with $\varepsilon<\delta$ by using (3.18). This completes the proof of (iii) and the proof of Theorem 3.1.

\section{COMPLETENESS THEOREM}

In order to discuss the wave front set of a hyperfunction it is necessary to consider analytic functionals carried by a compact set in $\mathbf{C}^{n}$.

Definition 4.1. Let $K$ be a compact set in $\mathbf{C}^{n}$. Then $A^{\prime}[K]$ is the space of linear forms $u$ on the space $A$ of entire analytic functions in $\mathbf{C}^{n}$ such that for every complex neighborhood $\omega$ of $K$

$$
|u(\phi)| \leq C_{\omega} \sup _{\omega}|\phi|, \quad \phi \in A .
$$

For $u \in A^{\prime}[K], K \Subset C^{n}$, we can also consider the defining function

$$
U(x, t)=u(E(x-\cdot, t)), \quad(x, t) \in R_{+}^{n+1} .
$$

We can see $U(\cdot, t) \rightarrow u$ as $t \rightarrow 0_{+}$in the following sense:

$$
u(\phi)=\lim _{t \rightarrow 0} \int_{\Omega} U(x, t) \phi(x) d x, \quad \phi \in A,
$$

where $\Omega \subset R^{n}$ is a suitably chosen neighborhood of $\operatorname{Re} K=\left\{x \in R^{n} ; x \in \operatorname{Re} z\right.$ for some $z \in K\}$. This is verified by virtue of (4.4) given in the following theorem.

Theorem 4.1 [3, Proposition 9.1.2]. Let $K \subset R^{n}$ be a compact set and set for $\varepsilon>0$

$$
K_{(\varepsilon)}=\left\{z \in \mathbf{C}^{n} ;|\operatorname{Re} z-x|+2|\operatorname{Im} z| \leq \varepsilon \text { for some } x \in K\right\} .
$$

For $\phi \in A$, set

$$
U^{\phi}(z, t)=\int_{\Omega} E(z-x, t) \phi(x) d x, \quad z \in C^{n}, t>0,
$$

where $\Omega \subset R^{n}$ is an arbitrary fixed bounded neighborhood of $\operatorname{Re} K_{(\varepsilon)}$. Then

$$
\sup _{K_{(\varepsilon)}}\left|U^{\phi}(\cdot, t)-\phi(\cdot)\right| \rightarrow 0 \quad \text { as } t \rightarrow 0_{+},
$$

and there exists a constant $C$ such that

$$
\sup _{K_{(\varepsilon)}}\left|U^{\phi}(\cdot, t)\right| \leq C \sup _{\bar{\Omega}_{(\varepsilon)}}|\phi|, \quad \phi \in A, t>0 .
$$

Proof. We shall reproduce the proof given in [3]. It is clear that $U^{\phi}(\cdot, t) \in A$ for $t>0$. By the definition of $K_{(\varepsilon)}$, if $z_{0}=x_{0}+i y_{0} \in K_{(\varepsilon)}$ it follows that $K_{(\varepsilon)}$ contains the chains

$$
\Gamma\left(z_{0}, s\right): x \rightarrow x+i y_{0}\left(1-\left|x-x_{0}\right| / 2\left|y_{0}\right|\right), \quad\left|x-x_{0}\right| \leq 2\left|y_{0}\right|,
$$


when $0 \leq s \leq 1$, and they have the same boundary. Since the form $E(z-\zeta, t) \phi(\zeta) d \zeta_{1} \wedge \cdots \wedge d \zeta_{n}$ is closed in $K_{(\varepsilon)}$ we obtain by Stokes' formula

$$
\begin{aligned}
U^{\phi}(z, t)= & \int_{\substack{\left|x-x_{0}\right|>2\left|y_{0}\right| \\
x \in \Omega}} E(z-x, t) \phi(x) d x \\
& +\int_{\Gamma\left(z_{0}, 1\right)} E(z-\zeta, t) \phi(\zeta) d \zeta_{1} \wedge \cdots \wedge d \zeta_{n} .
\end{aligned}
$$

We take $z=z_{0}$ and observe that

$$
\begin{aligned}
\operatorname{Re}-\left\langle z_{0}-x, z_{0}-x\right\rangle=-\left|x_{0}-x\right|^{2}+\left|y_{0}\right|^{2} \leq-3\left|x_{0}-x\right|^{2} / 4 & \\
& \text { if }\left|x-x_{0}\right|>2\left|y_{0}\right|, \\
\operatorname{Re}-\left\langle z_{0}-\zeta, z_{0}-\zeta\right\rangle=-\left|x_{0}-x\right|^{2}+\left|x-x_{0}\right|^{2} / 4 & =-3\left|x-x_{0}\right|^{2} / 4 \\
& \text { if } \zeta \in \Gamma\left(z_{0}, 1\right), \operatorname{Re} \zeta=x .
\end{aligned}
$$

Since $\left|\phi(\zeta)-\phi\left(z_{0}\right)\right| \leq C\left|x-x_{0}\right|$ we conclude that

$$
\begin{aligned}
& \left|U^{\phi}\left(z_{0}, t\right)-\phi\left(z_{0}\right) \int_{\Omega} E\left(z_{0}-x, t\right) d x\right| \\
& \quad \leq C_{0}(4 \pi t)^{-n / 2} \int_{\Omega} \exp \left[-3\left|x-x_{0}\right|^{2} / 4 t\right]\left|x-x_{0}\right| d x \leq C_{1} t^{1 / 2} .
\end{aligned}
$$

On the other hand

$$
1-\int_{\Omega} E\left(z_{0}-x, t\right) d x=\int_{R^{n} \backslash \Omega} E\left(z_{0}-x, t\right) d x
$$

is exponentially decreasing as $t \rightarrow 0$ since $\operatorname{Re}\left(z_{0}-x\right)^{2}$ has a positive lower bound when $z_{0} \in K_{(\varepsilon)}$ and $x \in R^{n} \backslash \Omega$. This implies (4.4). Estimate (4.5) is obvious by expression (4.5). Q.E.D.

We shall use the following notation similarly as before:

$$
A^{\prime}\left(\mathbf{C}^{n}\right)=\bigcup_{K \Subset C^{n}} A^{\prime}[K] .
$$

We prepare the following theorem corresponding to Theorem 2.3.

Theorem 4.2. If $K_{1}, \ldots, K_{r}$ are compact subsets of $R^{n}$ and

$$
u \in A^{\prime}\left[\left(K_{1} \cup \cdots \cup K_{r}\right)_{(\varepsilon)}\right]
$$

with the notation of (4.3), then one can find $u_{j} \in A^{\prime}\left[\left(K_{j}\right)_{(3 n \varepsilon)}\right]$ so that $u=$ $u_{1}+\cdots+u_{r}$, where $\varepsilon$ is an arbitrary fixed positive constant.

One of the main tools of the proof of Theorem 4.2 is the following fact due to Komatsu [5 and 7]: 
Lemma 4.1. For any $L>0$ and $\varepsilon>0$, there exist a function $v(t) \in C_{0}^{\infty}(R)$ and an ultradifferential operator $P(d / d t)$ such that

$$
\begin{gathered}
\operatorname{supp} v \subset[0, \varepsilon], \quad \begin{array}{c}
\left|v^{(k)}(t)\right| \leq C L^{-k} k !^{2}, \quad k=0,1, \ldots, \\
|v(t)| \leq C \exp [-L / t], \quad 0<t<\infty ;
\end{array} \\
p(d / d t)=\sum_{k=0}^{\infty} a_{k}(d / d t)^{k},\left|a_{k}\right| \leq C_{1} L_{1}^{k} / k !^{2}, \quad 0<L_{1}<L ; \\
P(d / d t) v(t)=\delta+w(t),
\end{gathered}
$$

where $w(t) \in C_{0}^{\infty}(R)$, supp $w \subset[\varepsilon / 2, \varepsilon]$.

Proof. We shall only give a sketch of the proof. First we set

$$
\begin{aligned}
& P(\zeta)=(1+\zeta)^{2} \prod_{q=1}^{\infty}\left(1+L \zeta / q^{2}\right) \quad(L>0), \\
& U(z)=\frac{1}{2 \pi i} \int_{0}^{\infty} e^{i z \zeta} P(\zeta)^{-1} d \zeta, \quad \operatorname{Re} z<0 .
\end{aligned}
$$

Then $U(z)$ is a holomorphic function which can be continued analytically to the Riemann domain $\{z ;-\pi<\arg z<2 \pi\}$. Furthermore, set

$$
u(x) \equiv U(x+i 0)-U(x-i 0) \text {. }
$$

Then we have

$$
\begin{gathered}
\left|u^{(q)}(x)\right| \leq \frac{1}{2} L^{-q} q !^{2}, \\
P(d / d x) u(x)=\delta(x), \\
u(x)=0 \text { for } x<0, \quad u(x) \geq 0 \text { for } x \geq 0 \text { and } \\
\int_{-\infty}^{\infty} u(x) d x=1 .
\end{gathered}
$$

Furthermore we have

$$
u(x) \leq \frac{1}{2} \exp [-L / x], \quad x>0 .
$$

The function $v(x)$ is obtained by multiplying $u(x)$ with a function in $\mathscr{E}^{(2)}(R)$ which is equal to 1 in $(-\infty, \varepsilon / 2]$ and equal to 0 in $[\varepsilon, \infty)$. By the definition of $P(\zeta)$ in (4.10) we can see that it is an entire function of order $1 / 2$ such that

$$
P(\zeta)=\sum_{k=0}^{\infty} a_{k} \zeta^{k}, \quad\left|a_{k}\right| \leq C_{1} L_{1}^{k} / k !^{2}, \quad 0<L_{1}<L .
$$

For the details of the proof we refer to [5 and 7].

Proof of Theorem 4.2. It is sufficient to prove the statement when $r=2$. Let $U(x, t)$ be the defining function of $u$ given by

$$
U(x, t)=u(E(x-\cdot, t)), \quad(x, t) \in R_{+}^{n+1} .
$$


The theorem will be proved if we can split into a sum $u=u_{1}+u_{2}$, where for any $\varepsilon^{\prime}>0$

$$
\left|u_{j}(\phi)\right| \leq C_{\varepsilon^{\prime}} \sup _{\left(K_{j}\right)_{\left(3 n+\varepsilon^{\prime}\right)}}|\phi|, \quad \phi \in A, j=1,2 .
$$

By the definition of $U$ in (4.18) we get the estimate

$$
\begin{array}{r}
|U(x, t)| \leq C_{\varepsilon^{\prime}} \exp \left[\left(\varepsilon^{2}-\operatorname{dis}\left(x, \operatorname{Re} K_{\left(\varepsilon+\varepsilon^{\prime}\right)}\right)^{2}\right) / 4 t\right], \quad K=K_{1} \cup K_{2}, \\
(x, t) \in R_{+}^{n+1} .
\end{array}
$$

Taking $L=\varepsilon^{2}$ in Lemma 4.1, we set

$$
g(x, t)=\int_{0}^{\infty} U(x, t+s) v(s) d s, \quad h(x, t)=\int_{0}^{\infty} U(x, t+s) w(s) d s .
$$

Then we have $g(x, t), h(x, t) \in L^{\infty}\left(R_{+}^{n+1}\right)$ and

$$
\begin{gathered}
P(d / d t) g(x, t)=U(x, t)+h(x, t), \quad(x, t) \in R_{+}^{n+1}, \\
(\partial / \partial t-\Delta) g(x, t)=0 \text { in } R_{+}^{n+1} .
\end{gathered}
$$

We can see that there are limits:

$$
\begin{aligned}
& g_{0}(x)=\lim _{t \rightarrow 0} g(x, t) \in L^{\infty}\left(R^{n}\right), \\
& h_{0}(x)=\lim _{t \rightarrow 0} h(x, t) \in L^{\infty}\left(R^{n}\right) \cap C^{\infty}\left(R^{n}\right) .
\end{aligned}
$$

Now let $\Omega$ be an arbitrary bounded neighborhood of $\operatorname{Re} K_{(\varepsilon)}$ with smooth boundary. Then we have

$$
\begin{aligned}
\int_{\Omega} P(d / d t) g(x, t) \phi(x) d x=\int_{\Omega} P(\Delta) g(x, t) \phi(x) d x \\
\quad=\int_{\Omega} U(x, t) \phi(x) d x+\int_{\Omega} h(x, t) \phi(x) d x, \quad \phi \in A, t>0 .
\end{aligned}
$$

We know that the first term in the last expression tends to $u(\phi)$ as $t \rightarrow 0_{+}$ by (4.2). We split $\Omega$ into a sum $\Omega=\Omega_{1}+\Omega_{2}$, where $\Omega_{1} \supset\left(K_{1}\right)_{(\varepsilon)}$ and $\Omega_{2} \supset\left(\operatorname{Re} K_{2}\right)_{(\varepsilon)} \backslash\left(\operatorname{Re} K_{1}\right)_{(\varepsilon)}$ with piecewise smooth boundaries. By applying partial integration in (4.22) we have

$$
\begin{aligned}
& \int_{\Omega} U(x, t) \phi(x) d x=\int_{\Omega} g(x, t) P(\Delta) \phi(x) d x \\
& +\int_{\partial \Omega} Q(g(x, t), \phi(x)) d S-\int_{\Omega} h(x, t) \phi(x) d x, \quad \phi \in A,
\end{aligned}
$$

where we set

$$
Q(u, v)=\sum_{k=1}^{\infty} a_{k} \sum_{j=0}^{k-1}\left[\Delta^{j} u \partial_{n}\left(\Delta^{k-1-j} v\right)-\Delta^{j} v \partial_{n}\left(\Delta^{k-1-j} u\right)\right] \text { on } \partial \Omega .
$$


We will put

$$
\begin{aligned}
u_{j}(\phi)= & \int_{\Omega_{j}} g_{0}(x) P(\Delta) \phi(x) d x+\int_{\partial \Omega \cap \partial \Omega_{j}} Q\left(g_{0}(x), \phi(x)\right) d S \\
& -\int_{\Omega_{j}} h_{0}(x) \Phi(x) d x, \quad j=1,2 .
\end{aligned}
$$

Obviously we have $u=u_{1}+u_{2}$ and we shall prove the estimates of the form (4.19). Now recall that we have $L_{1}<L=\varepsilon^{2}$ in Lemma 4.2 so that we have

$$
\left|a_{k}\right| \leq C_{1} L_{1}^{k} k !^{-2}, \quad k=0,1, \ldots
$$

Then we have for $\phi \in A$

$$
\begin{aligned}
\sup _{\Omega_{j}}|P(\Delta) \phi| & \leq \sup _{\Omega_{j}} \sum_{k=0}^{\infty}\left|a_{k} \Delta^{k} \phi(x)\right| \leq C_{1} \sum_{k=0}^{\infty} L_{1}^{k} k !^{-2} \sup _{\Omega_{j}}\left|\Delta^{k} \phi(x)\right| \\
& \leq C \sup _{K_{j},(3 n \varepsilon)}|\phi|, \quad j=1,2,
\end{aligned}
$$

where we have used Cauchy's integral formula and $C$ is a positive constant independent of $\phi \in A$. We see obviously

$$
\left|\int_{\Omega_{j}} h_{0}(x) \phi(x) d x\right| \leq C \sup _{\Omega_{j}}|\phi|, \quad \phi \in A, j=1,2 .
$$

It remains to estimate the second term in the right-hand side of (4.24). We recall that

$$
g_{0}(x)=\int_{0}^{\infty} U(x, s) v(s) d s, \quad x \in R^{n},
$$

and for $x \in \partial \Omega$ we have

$$
\begin{aligned}
\Delta^{j} g_{0}(x) & =\int_{0}^{\infty}(d / d s)^{j} U(x, s) v(s) d s \\
& =\int_{0}^{\infty} U(x, s)(-d / d s)^{j} v(s) d s, \quad j=1,2, \ldots
\end{aligned}
$$

Thus we have by (4.13)

$$
\left|\Delta^{j} g_{0}(x)\right| \leq C \varepsilon^{-2 j} j !^{2}, \quad j=0,1, \ldots .
$$

From this we find for $x \in \partial \Omega_{j} \cap \partial \Omega, j=1,2$,

$$
\left|Q\left(g_{0}(x), \phi(x)\right)\right| \leq C \sum_{k=1}^{\infty}\left(L_{1} / \varepsilon^{2}\right)^{k} \sum_{m=0}^{k}(m !(k-m) ! / k !)^{2} \sup _{\left(K_{j}\right)_{(2 n \varepsilon)}}|\phi|
$$

Since it holds that

$$
\sup _{k} \sum_{m=0}^{k}(m !(k-m) ! / k !)^{2}<\infty
$$

we have the estimate of the form

$$
\left|\int_{\partial \Omega_{j} \cap \partial \Omega} Q\left(g_{0}, \phi\right) d S\right| \leq C \sup _{K_{j,(2 n \varepsilon)}}|\phi|, \quad \phi \in A .
$$

Combining the above three estimates we get estimate (4.19). This completes the proof of Theorem 4.2. 
Theorem 4.3 [3, Theorem 9.1.7]. Let $K_{0}$ and $K$ be compact sets with $K_{0} \subset$ $K \subset R^{n}$, let $u_{j} \in A^{\prime}\left(\mathbf{C}^{n}\right)$ and assume that

(i) For any compact neighborhood $V$ of $K$ in $\mathbf{C}^{n}$ we have $u_{j} \in A^{\prime}[V]$ for large $j$.

(ii) For any compact neighborhood $V_{0}$ of $K_{0}$ we have $u_{j}-u_{k} \in A^{\prime}\left[V_{0}\right]$ for large $j, k$.

Then one can find $u \in A^{\prime}[K]$ so that for any compact neighborhood $V_{0}$ of $K_{0}$

(iii) $u-u_{j} \in A^{\prime}\left[V_{0}\right]$ for large $j$.

Condition (iii) determines $u$ uniquely $\bmod A^{\prime}\left[K_{0}\right]$.

Proof. If (iii) is fulfilled with $u$ replaced by $v$ also, then $u-v \in A^{\prime}\left[V_{0}\right]$ for every $V_{0}$ so $u-v \in A^{\prime}\left[K_{0}\right]$ as claimed. Thus it remains to prove the existence of $u$, which will be carried out by the method similar to that used in the proof of Theorem 2.4. Choose a sequence $\varepsilon_{j} \rightarrow 0$ so that with the notation in Theorem 4.1

(i) ${ }^{\prime} u_{j} \in A^{\prime}\left[K_{\left(e_{j}\right)}\right]$,

(ii) ${ }^{\prime} u_{j}-u_{k} \in A^{\prime}\left[\left(K_{0}\right)_{\left(e_{j}\right)}\right]$ if $k \geq j$.

Let $U_{j}(x, t)$ be the defining function of $u_{j}$ :

$$
U_{j}(x, t)=u_{j}(E(x-\cdot, t)), \quad(x, t) \in R_{+}^{n+1} .
$$

We shall show that there are functions $V_{j}(x, t)$ satisfying conditions (4.26)(4.31):

$$
(\partial / \partial t-\Delta) V_{j}(x, t)=0 \text { in } R_{+}^{n+1} .
$$

For any $\varepsilon>0$,

$$
\left|V_{j}(x, t)\right| \leq C_{j, \varepsilon} e^{\varepsilon / t} \text { in } R_{+}^{n+1} .
$$

For every $\delta>0$,

$$
\left|V_{j}(\cdot, t)\right| \rightarrow 0 \text { as } t \rightarrow 0_{+},
$$

uniformly in $\left\{x ; \operatorname{dis}\left(x, K_{0}\right) \geq \delta\right\}$; there are positive constants $C$ and $c$ such that

$$
\begin{aligned}
\left|U_{j+1}(x, t)-U_{j}(x, t)-V_{j}(x, t)\right| \leq C 2^{-j} \exp \left[c \varepsilon_{j} / t\right] \quad \text { in } R_{+}^{n+1}, \\
j=1,2, \ldots .
\end{aligned}
$$

For every $\delta>0$ there are positive constants $C_{\delta}, c_{\delta}$ and the number $j_{\delta}$ such that

$$
\left|U_{j+1}(x, t)-U_{j}(x, t)-V_{j}(x, t)\right| \leq C_{\delta} 2^{-j} \exp \left[-c_{\delta} / t\right]
$$

in $\left\{x \in R^{n} ; \operatorname{dis}\left(x, K_{0}\right) \geq \varepsilon_{j}+\delta\right\}$ for all $j \geq j_{\delta}$; for any $\varepsilon>0$ there exists a constant $C_{\varepsilon}$ such that

$$
\begin{aligned}
& \left|\int_{\Omega}\left(U_{j+1}(x, t)-U_{j}(x, t)-V_{j}(x, t)\right) \phi(x) d x\right| \\
& \quad \leq C_{\varepsilon} 2^{-j} \sup _{\left(K_{0}\right)\left(\varepsilon_{j}+\varepsilon\right)}|\phi|, \quad \phi \in A, t>0, j=1,2, \ldots,
\end{aligned}
$$


where $\Omega \subset R^{n}$ is an arbitrary fixed neighborhood of $K_{0}$ contained in $\operatorname{Re}\left(K_{0}\right)_{(\varepsilon)}$.

If these conditions are proved it follows from (4.29) that the limit

$$
\begin{aligned}
U(x, t) & =\lim _{j \rightarrow \infty}\left(U_{j}-V_{1}-\cdots-V_{j-1}\right) \\
& =U_{j}-V_{1}-\cdots-V_{j-1}+\sum_{j}^{\infty}\left(U_{k+1}-U_{k}-V_{k}\right)
\end{aligned}
$$

exists and $U(\cdot, t)$ defines $u=\lim _{t \rightarrow 0} U(\cdot, t) \in A^{\prime}[K]$ in the sense of (4.2). We see from (4.31) and (4.2) that $U-U_{j}$ defines $u-u_{j} \in A^{\prime}\left[\left(K_{0}\right)_{\left(e_{j}\right)}\right]$ which proves (iii). Thus $u$ is the desired element in $A^{\prime}[K]$.

We will construct such defining functions $V_{j}(x, t)$. As is easily seen, we may assume that $\varepsilon_{j}<1 / j, j=1,2, \ldots$. Then for each $j$ the compact region $\operatorname{Re} K_{\left(e_{j}\right)}$ can be covered by the finite number of balls with radius $2 / j$ and center on $\operatorname{Re} K_{\left(e_{j}\right)}$, say $B_{j_{k}}=\left\{x \in R^{n} ;\left|x-x_{j k}\right| \leq 2 / j, x_{j k} \in \operatorname{Re} K_{\left(e_{j}\right)}, k=1, \ldots, k_{j}\right\}$. Since $u_{j+1}-u_{j}$ is carried by $K_{e_{j}}$, we can split $u_{j+1}-u_{j}$ by Theorem 4.2 as follows:

$$
u_{j+1}-u_{j}=\sum_{k=1}^{k_{j}} g_{j k}
$$

where $g_{j k}$ is carried by $B_{j_{k},\left(3 n \varepsilon_{j}\right)}, k=1, \ldots, k_{j}$. Thus we have by (4.25)

$$
U_{j+1}(x, t)-U_{j}(x, t)=\sum_{k=1}^{k_{j}} g_{j k}(E(x-\cdot, t)) .
$$

By taking the Taylor expansion of $E$ at $x-x_{j k}$ we have

$$
g_{j k}(E(x-\cdot, t))=\sum_{\alpha} \frac{1}{\alpha !} \partial_{x}^{\alpha} E\left(x-x_{j k}, t\right) \int\left(x_{j k}-y\right)^{\alpha} g_{j k}(y) d y,
$$

where the integration is taken in the sense of distributions. We may suppose that there is a constant $C_{j}$ for each $j$ such that

$$
\left|\int g_{j k}(y)\left(x_{j k}-y\right)^{\alpha} d y\right| \leq C_{j}\left(4 n \varepsilon_{j}\right)^{|\alpha|} \leq C_{j}(4 n / j)^{|\alpha|}, \quad k=1, \ldots, k_{j} .
$$

For large $N$ determined later we set

$$
V_{j k}(x, t)=\sum_{|\alpha|<N} \frac{1}{\alpha !} \partial_{x}^{\alpha} E\left(x-x_{j k}, t\right) \int\left(x_{j k}-y\right)^{\alpha} g_{j k}(y) d y .
$$


Then we find by Proposition 1.1 that

$$
\begin{aligned}
(*) & \equiv\left|\int E(x-y, t) g_{j k}(y) d y-V_{j k}(x, t)\right| \\
& =\left|\sum_{|\alpha| \geq N+1} \frac{1}{\alpha !} \partial_{x}^{\alpha} E\left(x-x_{j k}, t\right) \int\left(x_{j k}-y\right)^{\alpha} g_{j k}(y) d y\right| \\
& \leq C \sum_{|\alpha| \geq N+1} C_{n}^{|\alpha|} t^{-(n+|\alpha|) / 2} \alpha !^{-1 / 2} \exp \left[-a\left|x-x_{j k}\right|^{2}\right](4 n / j)^{|\alpha|} .
\end{aligned}
$$

Taking $\varepsilon_{j}^{\prime}=\left(4 n C_{n}\right)^{2} / j^{2}$, we get

$$
(*) \leq C_{j}^{\prime} t^{-n / 2} \exp \left[\varepsilon_{j}^{\prime} / t-a\left|x-x_{j k}\right|^{2} / 4 t\right] \sum_{|\alpha| \geq N+1}(1 / 2)^{|\alpha|} .
$$

Thus if we take $N$ sufficiently large, we have

$$
\left|U_{j+1}(x, t)-U_{j}(x, t)-\sum_{k=1}^{k_{j}} V_{j k}\right| \leq 2^{-j} t^{-n / 2} \exp \left[\varepsilon_{j}^{\prime} / t\right], \quad \varepsilon_{j}^{\prime}=\left(4 n C_{n}\right)^{2} / j^{2} .
$$

If we set

$$
V_{j}(x, t)=\sum_{k=1}^{k_{j}} V_{j k}(x, t), \quad j=1,2, \ldots,
$$

assertions (4.26)-(4.30) are proved and we can easily verify (4.31) much as above. Q.E.D.

\section{WAVE FRONT SET}

Let $u \in A^{\prime}\left(R^{n}\right)$. Then the defining function of $u$ is given by

$$
U(x, t)=u(E(x-\cdot, t)), \quad(x, t) \in R_{+}^{n+1},
$$

and we may consider $U(\cdot, t) \in A$ for each $t>0$, where $A$ is the set of entire functions in $\mathbf{C}^{n}$. Theorem 3.1 suggests the following definition:

Definition 5.1. Let $u \in A^{\prime}\left(R^{n}\right)$. We denote by $\mathrm{WF}_{A}(u), \mathrm{WF}_{\{s\}}(u), 1<s<\infty$, and $\mathrm{WF}(u)$ respectively the complement in $T^{*}\left(R^{n}\right) \backslash 0$ of the set of $\left(x_{0}, \xi_{0}\right)$ such that there is a neighborhood $V$ of $x_{0}-i \xi_{0}$ in $\mathbf{C}^{n}$ and there are positive constants $C$ and $c$ such that

$$
\begin{gathered}
|U(z, t)| \leq C \exp \left[\xi_{0}^{2} / 4 t-c / t\right], \quad t>0, \quad z=x+i \xi \in V, \\
|U(z, t)| \leq C \exp \left[\xi^{2} / 4 t-(c / t)^{1 / s}\right], \quad t>0, \quad z \in V,
\end{gathered}
$$

and there are positive constants $C_{N}, N=0,1, \ldots$, such that

$$
|U(z, t)| \leq C_{N} t^{N} \exp \left[\xi^{2} / 4 t\right], \quad t \geq 0, z \in V,
$$

respectively. 
By this definition we have obviously the following inclusion:

$$
\mathrm{WF}(u) \subset \mathrm{WF}_{\{s\}}(u) \subset \mathrm{WF}_{A}(u), \quad u \in A^{\prime}\left(R^{n}\right), 1<s<\infty .
$$

From the expression

$$
\begin{aligned}
U(x+i \xi, t)= & (4 \pi t)^{-n / 2} \exp \left[\xi^{2} / 4 t-i\langle x, \xi\rangle / 2 t\right] \\
& \cdot u_{y}\left(\exp \left[-(x-y)^{2} / 4 t+i\langle y, \xi\rangle / 2 t\right]\right),
\end{aligned}
$$

we have the equivalent conditions to $(5.1),(5.1)_{\{s\}}$ and (5.1) respectively:

$$
\begin{aligned}
\left|u_{y}\left(\exp \left[-(x-y)^{2} / 4 t+i\langle y, \xi\rangle / 2 t\right]\right)\right| \leq C e^{-c / t}, & \\
t & >0, z=x+i \xi \in V,
\end{aligned}
$$

$(5.1)_{\{s\}}^{\prime}\left|u_{y}\left(\exp \left[-(x-y)^{2} / 4 t+i\langle y, \xi\rangle / 2 t\right]\right)\right| \leq C \exp \left[-(c / t)^{1 / s}\right]$,

$$
t>0, z \in V \text {, }
$$

$$
\left|u_{y}\left(\exp \left[-(x-y)^{2} / 4 t+i\langle y, \xi\rangle / 2 t\right]\right)\right| \leq C_{N} t^{N}, \quad t>0, z \in V .
$$

We also remark that for every $\varepsilon>0$ we always have the estimate of the form

$$
\begin{aligned}
|U(x+i \xi, t)| \leq C_{\varepsilon} \exp \left[\left(\varepsilon(1+|\xi|)+\xi^{2}-\operatorname{dis}(x, \operatorname{supp} u)^{2}\right) / 4 t\right] & \\
t & >0, z=x+i \xi \in C^{n}(\mathrm{cf} .(2.3)) .
\end{aligned}
$$

A direct application of Definition 5.1 is given to prove the following theorem (watermelon) originally due to [12]. A simple proof was given in [13] in case $u \in \mathscr{D}^{\prime}\left(R^{n}\right)$ whose method we shall adopt.

Theorem 5.1. Let $u \in A^{\prime}\left(R^{n}\right), \operatorname{supp} u \subset\left\{x_{n} \geq 0\right\}$. Then if $\left(0, \eta_{0}\right) \notin \mathrm{WF}_{A}(u)$ with $\eta_{0} \neq 0$, we have $\left(0,\left(\eta_{0}^{\prime}, \mu\right)\right) \notin \mathrm{WF}_{A}(u)$ for all $\mu \in R$.

Proof. Let $u \in A^{\prime}\left(R^{n}\right)$ with supp $u \subset\left\{x_{n} \geq 0\right\}$. Then we have by (5.3) an estimate of the type

$$
\begin{aligned}
& |U(x+i \xi, t)| \\
& \quad \leq \begin{cases}C_{\varepsilon} \operatorname{ext}\left[\left(\varepsilon(1+|\xi|)+\xi^{2}\right) / 4 t\right], & x_{n} \geq 0, \\
C_{\varepsilon} \exp \left[\left(\varepsilon(1+|\xi|)+\xi^{2}-x_{n}^{2}\right) / 4 t\right], & x_{n} \leq 0, t>0, x+i \xi \in C^{n} .\end{cases}
\end{aligned}
$$

Furthermore the assumption $\left(0, \eta_{0}\right) \notin \mathrm{WF}_{A}(u)$ implies that there exist positive constants $C$ and $c$ such that

$$
|U(x+i \xi, t)| \leq C \exp \left[\left(\eta_{0}^{2}-c\right) / 4 t\right]
$$

for $x+i \xi$ in a small neighborhood in $\mathbf{C}^{n}$ of $-i \eta_{0}$. Now the proof will be reduced to apply the next lemma to the function of a complex variable

$$
z_{n}=x_{n}+i \xi_{n} \mapsto \exp \left[-\xi_{0}^{\prime 2} / 4 t\right] U(x+i \xi, t), \quad t>0,
$$

for each $x^{\prime}+i \xi^{\prime}$ in a small neighborhood in $\mathbf{C}^{n-1}$ of $-i \eta_{0}^{\prime}$. 
Lemma 5.1. Let

$$
\phi(z)= \begin{cases}\xi^{2} / 2, & x \geq 0, \\ \left(\xi^{2}-x^{2}\right) / 2, & x \leq 0,\end{cases}
$$

where $z=x+i \xi \in C$ and let $R=\{z \in C ;|x| \leq a, 0 \leq \xi \leq b\} \quad(a, b>0)$. Then there exists a continuous function $\psi$ satisfying $\psi \leq \phi$ on $R$ and $\psi(i \xi)<$ $\phi(i \xi)$ for $0 \leq \xi \leq b$ so that the following assertion holds:

If $u(z)$ is holomorphic in $R$ and

$$
\begin{aligned}
|u(z)| \leq \exp [\lambda \phi(z)], & z \in R, \\
|u(z)| \leq \exp [\lambda(\phi(z)-c)], & z \in[-a, a],
\end{aligned}
$$

where $\lambda$ and $c$ are positive constants, then we have

$$
|u(z)| \leq \exp [\lambda \psi(z)] \text { in } R .
$$

Proof. With $0<\delta<a$ we apply the maximum principle in the rectangle $R_{\delta}=\{z ;-a+\delta<x<\delta, 0<\xi<b\}$ to

$$
v(x+i \xi)=\frac{1}{\lambda} \log |u(z)|-\left(\xi^{2}-x^{2}-\delta^{2}\right) / 2-c f(\xi) \sin \frac{\pi}{a}(\delta-x),
$$

where

$$
f(\xi)=(\exp [\pi b / a]-\exp [-\pi b / a])^{-1}(\exp [(-\pi / a)(\xi-b)-\exp [(\pi / a)(\xi-b)]) .
$$

Then we have $v(x+i \xi) \leq 0$ in $R$ and in particular

$$
\frac{1}{\lambda} \log |u(i \xi)|-\frac{1}{2} \xi^{2}<\delta^{2} / 2-c f(\xi) \sin (\pi \delta / a) .
$$

The right-hand side is negative in each compact interval in $[0 \leq \xi<b)$ if we take $\delta$ sufficiently small since $f(\xi)>0$ in $[0 \leq \xi<b)$. Q.E.D.

Theorem 5.2. Let $\Omega$ be a bounded open set in $R^{n}$. Let $\Gamma$ be an open convex cone in $R^{n} \backslash\{0\}$ and set for $\gamma>0$

$$
W=\left\{z \in \mathbf{C}^{n} ; \operatorname{Re} z \in \Omega, \operatorname{Im} z \in \Gamma,|\operatorname{Im} z|<\gamma\right\} .
$$

(i) If $f$ is an analytic function in $W$ and can be continued analytically to $a$ neighborhood of $\partial \Omega$, then the analytic functional

$$
\phi \rightarrow \int_{\Omega} f(x+i y) \phi(x) d x, \phi \in A,
$$

can be defined for sufficiently small $y \in \Gamma$ and converges in $A^{\prime}[\bar{\Omega}]$, when $\Gamma \ni$ $y \rightarrow 0$.

(ii) If $f$ is an analytic function in $W$ such that for any $\varepsilon>0$

(5.8) $|f(x+i y)| \leq C_{\varepsilon} \exp \left[(\varepsilon /|y|)^{1 /(s-1)}\right], \quad z=x+i y \in W,(1<s<\infty)$,

then $f(\cdot+i y)$ has a limit $f_{0} \in \mathscr{D}^{\{s\}^{\prime}}(\Omega)$ as $\Gamma \ni y \rightarrow 0$.

(iii) If $f$ is an analytic function in $W$ such that

$$
|f(x+i y)| \leq C|y|^{-N}, \quad z=x+i y \in W,
$$

then $f(\cdot+i y)$ has a limit $f_{0} \in \mathscr{D}^{\prime}(\Omega)$ as $\Gamma \ni y \rightarrow 0$. 
Remark. The uniqueness of the limit in this theorem will be proved in the more general situation given in the next theorem.

Proof of Theorem 5.2. (i) By assumption there is a small number $\varepsilon_{0}>0$ such that $f$ can be continued analytically to $(\partial \Omega)_{\left(e_{0}\right)}$. Choose $\chi \in C_{0}^{\infty}(\Omega)$ with $0 \leq \chi \leq 1$ so that $\chi=1$ on $\Omega \backslash\left((\partial \Omega)_{\left(\varepsilon_{0} / 2\right)} \cap R^{n}\right)$. Then for $y, \omega \in \Gamma,|y|<$ $\gamma,|\omega|<\gamma$,

$$
\int_{\Omega} f(x+i y) \phi(x) d x=\int_{\mu(\varepsilon, \omega)} f(z+i y) \phi(z) d z_{1} \wedge \cdots \wedge d z_{n}, \quad 0<\varepsilon<\varepsilon_{0},
$$

by Stokes' formula, $\mu(\varepsilon, \omega)$ denoting the chain

$$
\Omega \ni x \rightarrow x+i \varepsilon \chi(x) \omega .
$$

Letting $\Gamma \ni y \rightarrow 0$, this converges to

$$
F(\phi)=\int_{\mu(\omega, \varepsilon)} f(z) \phi(z) d z_{1} \wedge \cdots \wedge d z_{n}, \quad \phi \in A,
$$

which is independent of $\varepsilon, 0<\varepsilon<\varepsilon_{0}$. Furthermore for every $\varepsilon, 0<\varepsilon<\varepsilon_{0}$, there is a constant $C_{\varepsilon}>0$ such that

$$
|F(\phi)| \leq C_{\varepsilon} \sup _{\bar{\Omega}_{(\varepsilon)}}|\phi|, \quad \phi \in A .
$$

Thus we have $F \in A^{\prime}[\bar{\Omega}]$.

We remark that the uniqueness of $F$ with respect to $f$ in this case is easily showed by using Cauchy's integral formula for the chains $\mu(\varepsilon, \omega)$.

(ii) By the results on ultradifferentiable functions given in [5 and 7], we know that there exist a function $v \in \mathscr{D}^{\{s\}}(R)$ and an ultradifferential operator $P(d / d t)$ such that

$$
\begin{aligned}
& \operatorname{supp} v \subset[0, \gamma / 2) ; \\
& \exp \left[(\varepsilon / t)^{1 /(s-1)}\right] v(t) \in L^{\infty}(0, \infty) \quad \text { for any } \varepsilon>0
\end{aligned}
$$

(5.13) $P(d / d t) v(t)=\delta+w(t), \quad w(t) \in C_{0}^{\infty}(R), \operatorname{supp} w \subset[\gamma / 4, \gamma / 2)$.

We consider the function $g(x, t)$ defined by

$$
g(x, t)=\int_{0}^{\infty} f(x+i(s+t) y) v(s) d x, \quad y \in \Gamma .
$$

Then $g(x, t) \in L^{\infty}(\Omega \times[0<t<\gamma / 2))$ and there exists

$$
\lim _{t \rightarrow 0} g(\cdot, t)=\int_{0}^{\infty} f(x+i s y) v(s) d s \equiv g_{0} \in L^{\infty}(\Omega) .
$$

Furthermore we have for $(x, t) \in \Omega \times[0, \gamma / 2)$

$$
\begin{aligned}
P(d / d t) g(x, t) & =P\left(i \sum_{j=1}^{n} y_{j} \partial / \partial x_{j}\right) g(x, t) \\
& =f(x+i t y)+\int_{0}^{\infty} f(x+i(s+t) y) w(s) d s .
\end{aligned}
$$


Thus we have for $\phi \in \mathscr{D}^{\{s\}}(\Omega)$

$$
\begin{aligned}
\int_{\Omega} g(x, t) P\left(-i \sum_{j=1}^{n} y_{j} \partial / \partial x_{j}\right) \phi(x) d x \\
\quad=\int_{\Omega} f(x+i t y) \phi(x) d x+\int_{\Omega}\left(\int_{0}^{\infty} f(x+i(s+t) y) w(s) d s\right) \phi(x) d x,
\end{aligned}
$$

from where we find that $\int_{\Omega} f(x+i t y) \phi(x) d x$ converges to

$$
\int_{\Omega} g_{0}(x) P\left(-i \sum_{j=1}^{n} y_{j} \partial / \partial x_{j}\right) \phi(x) d x-\int_{\Omega}\left(\int_{0}^{\infty} f(x+i s y) w(s) d s\right) \phi(x) d x
$$

as $t \rightarrow 0$. We can easily see that $P\left(-i \sum_{j=1}^{n} y_{j} \partial / \partial x_{j}\right)$ is an ultradifferential operator in $\mathscr{D}^{\{s\}}(\Omega)$ since $P(d / d t)$ is an ultradifferential operator in $\mathscr{D}^{\{s\}}(R)$.

(iii) Take a function $v(t)$ such that

$$
v(t)= \begin{cases}t^{N} / N !, & 0<t<\gamma / 4 \\ 0, & t \leq 0 \text { and } \gamma / 2 \leq t<\infty\end{cases}
$$

Then we have

$$
\left(d^{N+1} / d t^{N+1}\right) v(t)=\delta+w(t)
$$

where $w(t) \in C_{0}^{\infty}(R)$ and supp $w \subset[\gamma / 4, \gamma / 2]$. By making use of this function we can prove the existence of $\lim _{t \rightarrow 0} f(\cdot+i t y) \in \mathscr{D}^{\prime}(\Omega)$ as in the proof of part (ii) for any fixed $y \in \Gamma$. Q.E.D.

Theorem 5.3 (cf. [3, Theorem 9.3.3]). Let $W=\{x+i y ; x \in \Omega, y \in \Gamma,|y|<\gamma\}$ be the same as in Theorem 5.2. Let $f$ be an analytic function in $W$. Then

(i) for any $\Omega_{1} \Subset \Omega$, there is an element $f_{\Omega_{1}} \in A^{\prime}[\bar{\Omega}]$ uniquely determined $\bmod A^{\prime}[\partial \Omega]$ such that the analytic functional

$$
A \ni \phi \rightarrow f_{\Omega_{1}}(\phi)-\int_{\Omega_{1}} f(x+i y) \phi(x+i y) d x
$$

is carried by any given neighborhood of $\partial \Omega_{1}$ in $C^{n}$ when $y \in \Gamma$ and $y$ is small enough. Thus $f_{\Omega_{1}}$ defines uniquely a hyperfunction in $B\left(\Omega_{1}\right)$.

(ii) If $\Omega_{2} \subset \Omega_{1}$ is another open set then $f_{\Omega_{1}}-f_{\Omega_{2}} \in A^{\prime}\left[\bar{\Omega}_{1} \backslash \Omega_{2}\right]$ so there is a unique $F \in B(\Omega)$ such that $F$ and $f_{\Omega_{1}}$ have the same restriction to $\Omega_{1}$ for every $\Omega_{1} \Subset \Omega$.

(iii) The limit in each case of (i), (ii) and (iii) in Theorem 5.2 is equal to $F$ on $\Omega$.

(iv) If $F=0$ in some nonempty $\Omega_{1} \subset \Omega$ and $W$ is connected, then $f=0$. Proof. (i) Let $\Omega_{0} \Subset \Omega$. For any $y_{1}, y_{2} \in \Gamma,\left|y_{1}\right|,\left|y_{2}\right|<\gamma$, we can easily see that the analytic functional

$$
A \ni \phi \rightarrow \int_{\Omega_{0}} f\left(x+i y_{1}\right) \phi\left(x+i y_{1}\right) d x-\int_{\Omega_{0}} f\left(x+i y_{2}\right) \phi\left(x+i y_{2}\right) d x
$$


is carried by $\partial \Omega_{0}+i\left[y_{0}, y_{1}\right]$. The existence of $f_{\Omega_{1}} \in A^{\prime}\left[\bar{\Omega}_{0}\right]$ is thus a consequence of Theorem 4.3.

(ii) Since $\int_{\Omega_{1} \backslash \Omega_{2}} f(x+i y) \phi(x+i y) d x$ is carried by $\left(\bar{\Omega}_{1} \backslash \Omega_{2}\right)+i y$, we find that $f_{\Omega_{1}}-f_{\Omega_{2}}$ is carried by any neighborhood of $\bar{\Omega}_{1} \backslash \Omega_{2}$, which means $f_{\Omega_{1}}-f_{\Omega_{2}} \in$ $A^{\prime}\left[\bar{\Omega}_{1} \backslash \Omega_{2}\right]$.

(iii) Let $f_{0} \in A^{\prime}[\bar{\Omega}]$ be the limit in (i) in Theorem 5.2. If we observe that $\phi(x+i y) \rightarrow \phi(x)$ in $A[\bar{\Omega}]$ as $y \rightarrow 0$, we have

$$
\begin{aligned}
f_{0}(\phi) & =\lim _{\Gamma \ni y \rightarrow 0} \int_{\Omega} f(x+i y) \phi(x) d x \\
& =\lim _{\Gamma \ni y \rightarrow 0} \int_{\Omega} f(x+i y) \phi(x+i y) d x, \quad \phi \in A .
\end{aligned}
$$

Next, let $f_{0} \in \mathscr{D}^{\{s\}^{\prime}}(\Omega)$ be the limit in (ii) in Theorem 5.2. Let $\Omega_{1} \in \Omega$ and $f_{0, \Omega_{1}}$ be the restriction of $f_{0}$ to $\Omega_{1}$. Choose $\phi \in \mathscr{D}^{\{s\}}(\Omega)$ so that $\phi=1$ in a neighborhood of $\bar{\Omega}_{1}$. Then for $\psi \in A$ we have by (5.15)

$$
\begin{aligned}
\int_{\Omega} f(x+i t y) \phi(x) \psi(x+i t y) d x \\
=\int_{\Omega} g(x, t) P\left(-i \sum_{j=1}^{n} y_{j} \partial / \partial x_{j}\right) \phi(x) \psi(x+i t y) d x \\
\quad-\int_{\Omega}\left(\int_{0}^{\infty} f(x+i(s+t) y) w(s) d s\right) \phi(x) \psi(x+i t y) d x .
\end{aligned}
$$

If we integrate only over $\Omega_{1}$ and letting $t \rightarrow 0$, we see $f_{\Omega_{1}}-f_{0, \Omega_{1}}$ is carried by any neighborhood of $\partial \Omega_{1}$. This means $f_{\Omega}=f_{0}$ in $\Omega$. The proof in case $f_{0} \in \mathscr{D}^{\prime}(\Omega)$ is similar, we omit it here.

(iv) We denote by $R_{y}$ the difference in (i). By assumption $R_{y}$ is carried by any neighborhood of $\partial \Omega_{1}$ when $|y|$ is small. We have

$$
f_{\Omega_{1}}(E(z-\cdot, t))-\int_{\Omega_{1}} E(z-\xi-i y, t) f(\xi+i y) d \xi=R_{y}(E(z-\cdot, t)),
$$

Taking $z=x+i y$ and letting $t \rightarrow 0$, we see $f(x+i y)$ is equal to zero for $x \in \Omega_{1}$ when $|y|$ is small, so $f=0$ in $W$ if $W$ is connected, which proves (iii). Q.E.D.

We call the hyperfunction $f_{\Omega} \in B(\Omega)$ the boundary value of $f$ from $\Gamma$ following [3]. We shall use the notation $b_{\Gamma}(f)=f_{\Omega}$ when we want to emphasize that the limit is taken from $\Gamma$ and $f$ is called $\Gamma$-analytic at $\Omega$.

Theorem 5.4 (cf. [13, Theorem 6.5]). Let $u \in A^{\prime}\left(R^{n}\right)$ and $\left(x_{0}, \xi_{0}\right) \in T^{*}\left(R^{n}\right) \backslash 0$. Then

(i) $\left(x_{0}, \xi_{0}\right) \notin \mathrm{WF}_{A}(u)$ if and only if there are open convex cones $\Gamma_{1}, \ldots$, $\Gamma_{N} \subset\left\{\xi \in R^{n} ;\left\langle\xi, \xi_{0}\right\rangle<0\right\}$, bounded open neighborhood $Z$ of $x_{0}$ in $\mathbf{C}^{n}, u_{0}$ 
holomorphic in $Z, f_{j}$ holomorphic in $Z \cap\left\{R^{n}+i \Gamma_{j}\right\}, j=1, \ldots, N$, so that

$$
u=u_{0}+\sum_{1}^{N} b_{\Gamma_{j}}\left(f_{j}\right) \text { on } Z \cap R^{n}
$$

(ii) $\left(x_{0}, \boldsymbol{\xi}_{0}\right) \notin \mathrm{WF}_{\{s\}}(u)(1<s<\infty)$ if and only if $u_{0} \in \mathscr{E}^{\{s\}}\left(Z \cap R^{n}\right)$ in the above expression (5.16).

(iii) $\left(x_{0}, \xi_{0}\right) \notin \mathrm{WF}(u)$ if and only if $u_{0} \in \mathscr{E}\left(Z \cap R^{n}\right)$ in the above expression (5.16).

Proof. (i) Let $u \in A^{\prime}\left(R^{n}\right)$. Then by (3.10) we know for $\left|x-x_{0}\right|<\varepsilon$ with $\varepsilon>0$ sufficiently small

$$
\begin{aligned}
& u(x)=(2 \pi)^{-n} \iint_{\substack{\left|\beta-x_{0}\right| \leq 2 \varepsilon \\
|\xi| \geq 1}} u_{y}\left(\exp \left[-i\langle x-y, \xi\rangle-|\xi|(\beta-y)^{2} / 2\right]\right) \\
& \cdot(|\xi| / 2 \pi)^{n / 2} d \xi d \beta+w_{\varepsilon}(x),
\end{aligned}
$$

where $w_{\varepsilon}(x)$ is analytic in $\left\{\left|x-x_{0}\right|<\varepsilon\right\}$. Now assume that $\left(x_{0}, \xi_{0}\right) \notin$ $\mathrm{WF}_{A}(u)$, then we see by $(5.1)^{\prime}$ there is a small cone neighborhood $V$ of $\xi_{0}$ in $R^{n} \backslash 0$ so that the following estimation holds:

$$
\left|\int_{\left|\beta-x_{0}\right| \leq 2 \varepsilon} u_{y}\left(\exp \left[i\langle x-y, \xi\rangle-|\xi|(\beta-y)^{2} / 2\right]\right)(|\xi| / 2 \pi)^{n / 2} d \beta\right| \leq C e^{-c|\xi|},
$$

$\boldsymbol{\xi} \in V$,

where $C$ and $c$ are positive constants independent of $x \in R^{n},\left|x-x_{0}\right| \leq \varepsilon$ and $\xi \in V$. For $\xi_{1} \in R^{n} \backslash 0,\left\langle\xi_{1}, \xi_{1}\right\rangle<0$, let $\Gamma_{\xi_{1}}$ be a closed cone neighborhood of $\xi_{1}$ such that

$$
\left\langle\eta, \xi_{1}\right\rangle>0,\left\langle\eta, \xi_{0}\right\rangle<0 \text { for any } \eta \in \Gamma_{\xi_{1}} .
$$

Let $\Gamma_{\xi_{1}}^{*}$ be the dual cone of $\Gamma_{\xi_{1}}$, i.e.

$$
\langle\eta, \xi\rangle>0 \text { on } \Gamma_{\xi_{1}} \times \Gamma_{\xi_{1}}^{*} \text {. }
$$

We can choose $\xi_{1}, \ldots, \xi_{N}$ so that

$$
R^{n} \backslash 0=V \cup \stackrel{\circ}{\Gamma}_{\xi_{1}}^{*} \cup \cdots \cup \stackrel{\circ}{\Gamma}_{\xi_{N}}^{*} .
$$

Then we have for $\left|x-x_{0}\right|<\varepsilon$

$$
\begin{aligned}
u(x) & =u_{0}+\sum_{1}^{N}(2 \pi)^{-n} \iint_{\substack{\left|\beta-x_{0}\right| \leq 2 \varepsilon \\
|\xi| \geq 1}} u_{y}\left(\exp \left[i\langle x-y, \xi\rangle-|\xi|(\beta-y)^{2} / 2\right]\right) \\
& \cdot(|\xi| / 2 \pi)^{n / 2} \chi_{j}(\xi /|\xi|) d \xi d \beta \\
& =u_{0}(x)+\sum_{1}^{N} u_{j}(x),
\end{aligned}
$$


where supp $\chi_{j} \subset \stackrel{\circ}{\Gamma}_{\xi_{j}}^{*}, \sum_{1}^{N} \chi_{j}=1$ on $R^{n} \backslash V$. It is easy to see that $u_{0}(x)$ is analytic in $\left|x-x_{0}\right|<\varepsilon$ and $u_{j}$ is the boundary value of

$$
\begin{array}{r}
f_{j}(z)=(2 \pi)^{-n} \iint_{\substack{\left|\beta-x_{0}\right| \leq 2 e \\
|\xi| \geq 1}} u_{y}\left(\exp \left[i\langle z-y, \xi\rangle-|\xi|(\beta-y)^{2} / 2\right]\right) \\
\cdot(|\xi| / 2 \pi)^{n / 2} \chi_{j}(\xi /|\xi|) d \xi d \beta
\end{array}
$$

which is analytic in $\tilde{Z} \cap\left(R^{n}+i \Gamma_{\xi_{j}}\right)$ with a small complex neighborhood $\tilde{Z}$ of $x_{0}$.

Conversely, assume (5.16). It is sufficient to prove the case $N=1$. Let $\Gamma$ be an open convex cone in $\left\{\xi \in R^{n} ;\left\langle\xi, \xi_{0}\right\rangle<0\right\}$ and let $Z$ be a complex neighborhood of $x_{0} \in R^{n}$. We assume $f$ is holomorphic in $Z \cap\left(R^{n}+i \Gamma\right)$ and $u \in A^{\prime}\left(R^{n}\right), u=b_{\Gamma}(f)$ on $Z \cap R^{n}$ whose existence is assured by Theorem 5.3 and Theorem 4.3. Take $\Omega=\left\{x \in R^{n} ;\left|x-x_{0}\right|<\delta\right\}$ so that $\bar{\Omega} \subset Z \cap R^{n}$ and write $u=u_{1}+u_{2}$ where $\operatorname{supp} u_{1} \subset \bar{\Omega}$ and $\operatorname{supp} u_{2} \subset R^{n} \backslash \Omega$. Let $U_{1}(x, t)$ and $U_{2}(x, t)$ be the defining functions of $u_{1}$ and $u_{2}$ respectively. By (5.3) we have the estimation of the form

$$
\left|U_{2}(x+i \xi, t)\right| \leq C \exp \left[\left(\xi^{2}-c\right) / 4 t\right], \quad \xi \in R^{n}, t>0,
$$

if $x$ is in a small neighborhood of $x_{0}$ contained in $\Omega$ and $\xi$ is any bounded set of $R^{n}$. For $y \in \Gamma$ with $|y|$ sufficiently small, we can see that the analytic functional

$$
A \ni \phi \rightarrow u_{1}(\phi)-\int_{\Omega} f(x+i y) \phi(x+i y) d x
$$

is carried by $\partial \Omega+[0, i y]$. Hence $u_{1}$ is carried by

$$
M=(\partial \Omega+[0, i y]) \cup(\bar{\Omega}+\{i y\}) .
$$

Recall that

$$
U_{1}(x+i \xi, t)=u_{1}(E(x+i \xi-\cdot, t)) .
$$

We can easily see that if we take $|y|$ sufficiently small there is a small neighborhood $V\left(x_{0}, \xi_{0}\right)$ of $\left(x_{0}, \xi_{0}\right)$ such that for $\mu+i \gamma$ near to $M$ we have

$$
\begin{aligned}
-\operatorname{Re}(x+i \xi-\mu-i \gamma)^{2} & =-(x-\mu)^{2}+(\xi-\gamma)^{2} \\
& \leq \xi_{0}^{2}-c, \quad(x, \xi) \in V\left(x_{0}, \xi_{0}\right),
\end{aligned}
$$

where $c>0$ is independent of $(x, \xi) \in V\left(x_{0}, \xi_{0}\right)$. Thus for the defining function $U$ of $u$ we have the estimate of the form

$$
|U(x-i \xi, t)| \leq C \exp \left[\left(\xi_{0}^{2}-c\right) / 4 t\right], \quad(x, \xi) \in V\left(x_{0}, \xi_{0}\right),
$$

which proves $\left(x_{0}, \xi_{0}\right) \notin \mathrm{WF}_{A}(u)$.

The proof of parts (ii) and (iii) is similar and we omit it here. 


\section{REFERENCES}

1. I. M. Gel'fand and G. E. Shilov, Generalized functions, vol. 2, Academic Press, New York and London, 1968.

2. L. Hörmander, Linear partial differential operators, Springer-Verlag, Berlin and New York, 1963.

3. _. The analysis of linear partial differential operators, vol. 1, Springer-Verlag, Berlin and New York, 1983.

4. D. Iagolnitzer, Microlocal essential support of distribution and decomposition theorems-an introduction, Hyperfunctions and Theoretical Physics, Lecture Notes in Math., vol. 449, Springer-Verlag, Berlin and New York, 1975, pp. 121-132.

5. H. Komatsu, Ultradistributions. I, Structure theorems and a characterization, J. Fac. Sci. Univ. Tokyo Sect. IA Math. 20 (1973), 25-105.

6. __ Ultradistributions. II, The kernel theorem and ultradistributions with support in a submanifold, J. Fac. Sci. Univ. Tokyo Sect. IA 24 (1977), 607-628.

7. __ Introduction to the theory of distributions (Japanese), Iwanami Shoten, Tokyo, 1978.

8. A. Martineau, Les hyperfonctions de M. Sato, Sém. Bourbaki 1960-1961, Exposé No. 214.

9. T. Matsuzawa, A calculus approach to hyperfunctions. I, Nagoya Math. J. 108 (1987), 53-66.

10. _ Gevrey hypoellipticity of a class of pseudodifferential operators, Tôhoku Math. J. 39 (1987), 447-464.

11. T. Matsuzawa, Hypoellipticity in ultradistribution spaces, J. Fac. Sci. Univ. Tokyo Sect. IA 34 (1987), 779-790.

12. M. Sato, T. Kawai and M. Kashiwara, Hyperfunctions and pseudodifferential equations, Lecture Notes in Math., vol. 287, Springer-Verlag, Berlin and New York, 1973, pp. 265-529.

13. J. Sjöstrand, Singularités analytiques microlocales, Astérisque 95 (1982), 1-166.

14. _. Propagation of analytic singularities for second order Dirichlet problems, Comm. Partial Differential Equations 5 (1980), 41-94.

Department of Mathematics, Faculty of Science, Nagoya University, Chikusa-ku, NAGOYA, 464 JAPAN 